\title{
PROGRESS IN ELECTROWEAK BARYOGENESIS
}

To appear in: Annual Review of Nuclear and Particle Science, vol. 43

\author{
A. G. Cohen ${ }^{a}$ \\ Physics Department \\ Boston University \\ Boston, MA 02215 \\ D. B. Kaplan ${ }^{b} c$ and A. E. Nelson $b d$ \\ Department of Physics 0319 \\ University of California, San Diego \\ 9500 Gilman Drive \\ La Jolla, CA 92093-0319
}

UCSD-PTH-93-02, BUHEP-93-4

January 1993

Email: cohen@andy.bu.edu, dkaplan@ucsd.edu, anelson@ucsd.edu

$a$ DOE Outstanding Junior Investigator

$b$ Sloan Fellow

${ }^{c}$ NSF Presidential Young Investigator

$d$ SSC fellow 


\section{TABLE OF CONTENTS}

1. INTRODUCTION . . . . . . . . . . . . . . . . . . . . . . . . . . 2

2. HIGH TEMPERATURE ELECTROWEAK BARYON VIOLATION . . . . . . 6

2.1. Baryon Violation and the Anomaly . . . . . . . . . . . . . . . . . . . 6

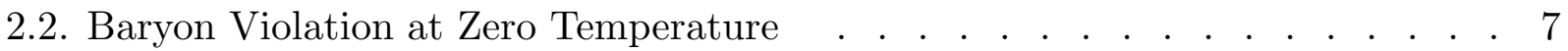

2.3. Baryon Violation at non-Zero Temperature $\quad . \quad$. . . . . . . . . . . . . . . . 9

2.4. Rates . . . . . . . . . . . . . . . . . . . . . . . . . . . 12

2.5. Baryon Violation at the Phase Transition . . . . . . . . . . . . . . . . 15

3. THE WEAK PHASE TRANSITION $\quad . \quad$. . . . . . . . . . . . . . . . . 16

3.1. The Nature of the Transition . . . . . . . . . . . . . . . . . . . . . 16

3.2. Dynamics of the Transition in the Early Universe . . . . . . . . . . . . . 22

3.3. Propagation and Shape of the Bubbles . . . . . . . . . . . . . . . . . 23

4. CALCULATING THE BARYON ASYMMETRY . . . . . . . . . . . . . . . . 26

4.1. $C P$ violation . . . . . . . . . . . . . . . . . . . . . . . . . . . . 26

4.2. Timescales . . . . . . . . . . . . . . . . . . . . . . . . . . . . . 28

4.3. The adiabatic "thick wall" regime: spontaneous baryogenesis . . . . . . . 29

4.4. The nonadiabatic "thin wall" regime:

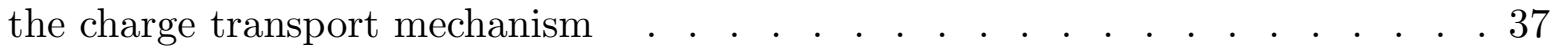

4.5. Conclusions about mechanisms . . . . . . . . . . . . . . . . . . . . . 41

5. CONSTRAINTS AND EXPERIMENTAL SIGNATURES ． . . . . . . . . . 41

6. FUTURE CALCULATIONS . . . . . . . . . . . . . . . . . . . . . . . 43

REFERENCES . . . . . . . . . . . . . . . . . . . . . . . . 44

FIGURE CAPTIONS . . . . . . . . . . . . . . . . . . . . . . . . . . 50 


\section{INTRODUCTION}

In the standard hot big bang model, relics from the early universe can give us much useful information about microphysics. For instance the abundances of the light elements, produced when the universe was at a temperature of $\sim 1 \mathrm{MeV}$, told us long before the existence of LEP that there were at most four, and probably three, species of neutrinos [1]. The most obvious of big bang relics are the baryons, which make our own existence possible. Furthermore, the universe seems to contain relatively few antibaryons. There is clear evidence that at least the local cluster of galaxies is made of matter, and there is no plausible mechanism to separate matter from antimatter on such large scales. The observed abundance of baryons today implies that when the universe was much hotter than a $\mathrm{GeV}$ the ratio of antibaryons to baryons must have been about one part in $10^{8}$ [1]. Sakharov pointed out in 1967 [2] that this cosmological asymmetry between matter and antimatter could be a calculable result of particle interactions in the early universe, teaching us several profound things about fundamental physics:

1) The number of baryons is not conserved.

2) $C$ (charge conjugation symmetry) and $C P$ (the product of charge conjugation symmetry and parity) are not exact symmetries.

3) The universe must have been out of thermal equilibrium in order to produce net baryon number, since the numbers of baryons and of antibaryons are equal in thermal equilibrium. Note that all known interactions are in thermal equilibrium when the temperature of the universe is between $100 \mathrm{GeV}$ and $10^{12} \mathrm{GeV}$.

Sakharov suggested that baryogenesis took place immediately after the big bang, at a temperature not far below the Planck scale of $10^{19} \mathrm{GeV}$, when the universe was expanding so rapidly that many processes were out of thermal equilibrium. This scenario was explicitly realized with the advent of grand unified theories (GUTs) [3-9], which predict baryon number violation, and possibly $C P$ violation, mediated by ultra-heavy particles [10].

This "standard" GUT baryogenesis scenario is appealingly simple but does not easily fit into an acceptable cosmology. One difficulty is that in the standard model of electroweak interactions baryon number is known theoretically to be anomalous and not exactly conserved [11]. At low temperature this anomalous baryon violation only proceeds via an exponentially suppressed tunnelling process, at a rate proportional to $\exp \left(-4 \pi / \alpha_{w}\right) \sim 0$. At temperatures above $\sim 100 \mathrm{GeV}$, however, electroweak baryon violation may proceed 
rapidly enough to equilibrate to zero any baryons produced by grand unification [4, 12]. In the last decade there has been much work indicating that this is the case 13 20. However anomalous electroweak processes conserve $B-L$, the difference between baryon and lepton numbers, and so a net $B-L$ generated by grand unified or other interactions will not be erased by electroweak interactions. In the minimal standard model if $B-L$ is non zero then the equilibrium baryon number at high temperature is $B=(12 / 37)(B-L)$ [15, $21-26$ 1 1 . The possibility that the observed baryons are a relic from a net $B-L$ generated at the GUT scale would be constrained if new $B-L$ violating physics (such as Majorana neutrino masses) were discovered [24, 25, 27-29].

Another problem with GUTs is that they predict a relic abundance of massive stable magnetic monopoles much larger than the observed matter density [30, 31]. The simplest GUT models which have an experimentally acceptable weak mixing angle are supersymmetric [32, 33], and in these models the relic abundance of gravitinos (supersymmetric partners of the graviton) is incompatible with constraints on the mass density of the universe 34 39]. The most attractive solution to these problems, which also explains the flatness and homogeneity of the observed universe, is inflation 40,42, which greatly dilutes the abundances of unwanted relics. However after an inflationary epoch any remaining asymmetry between the numbers of baryon and antibaryons is negligible, and the universe typically reheats to a temperature which is less than or of order $10^{12} \mathrm{GeV}$, well below the scale of grand unification [1]. Furthermore gravitino decays may affect nucleosynthesis [37, 38 unless the reheat temperature is below $10^{9} \mathrm{GeV}$, a temperature too cool to reinstate the baryon asymmetry through GUT processes.

There have also been many explanations of the matter-antimatter asymmetry which do not rely on GUTs [26]. Typically these propose the existence of new interactions and particles which exist solely in order to create baryons, and which are otherwise unmotivated and experimentally untestable.

In 1985 Kuzmin, Rubakov \& Shaposhnikov suggested an elegant and simple solution to the baryogenesis problem [15]. They argued that anomalous baryon number violation in the standard model of electroweak interactions is rapid at high temperatures and that the weak phase transition [43], if first order with supercooling, provides a natural way for the universe to get out of thermal equilibrium at weak scale temperatures. Eventually bubbles

1 There has been some confusion in the literature over this calculation; the correct fraction is found in [25, 26]. 
of the broken phase nucleate and expand until they fill the universe; local departure from thermal equilibrium takes place in the vicinity of the expanding bubble walls. Since $C$ and $C P$ are known to be violated by the electroweak interactions, it is possible to satisfy all the Sakharov baryogenesis conditions within the standard model. This suggestion opened up the exciting possibility that we may be able to compute the baryon number of the universe in terms of experimentally measurable aspects of the weak interactions, such as particle masses and $C P$ violating parameters.

The effects of the $C P$ violation in the minimal standard model from the KobayashiMaskawa (KM) phase [44] are much too small to explain the observed baryon to entropy ration. Explaining this number via baryogenesis in the early universe would require a $C$ and $C P$ violating asymmetry larger than $10^{-8}$ in some out of equilibrium reaction, while at high temperature $C P$ violation from the KM phase is always multiplied by a function of small couplings and mixing angles of order $10^{-20}$ 45, 47]. For example, in minimal SU(5) GUT-scale baryogenesis with only KM $C P$ violation one finds a baryon to photon ratio $n_{B} / n_{\gamma} \simeq 10^{-20}$ [9]. However the feasibility of baryogenesis during the electroweak phase transition has been demonstrated in several simple non-minimal versions of the standard model 48-55]. Furthermore the baryon asymmetry of the early universe can be used to constrain parameters in such non-minimal models; for instance in the singlet majoron model [56] an acceptable baryon abundance is obtainable only if the mass of the $\tau$ neutrino is greater than $5 \mathrm{MeV} 48$.

A crucial test of weak scale baryogenesis comes from the requirement that any baryons produced during the transition should survive until the present. If anomalous baryon violating processes occur at a rate which is faster than the expansion rate of the universe, then thermal and chemical equilibrium will be restored after the transition. Since the equilibrium abundances of baryons and antibaryons are equal, any baryon number which was created during the transition could be washed out. In the broken phase, the rate of baryon number violation is computed to be proportional to $\exp (-\mathcal{O}(1) 4 \pi v / g T)$, where $v$ is the value of the order parameter, $g$ is the weak coupling constant and $T$ is the temperature immediately after the transition [15, 16]. Thus suppression of the anomalous baryon

2 Shaposhnikov has suggested two conceivable ways to enhance the $C P$ violation in the standard model at high temperature [45]; the first mechanism, dynamical high temperature spontaneous $C P$ violation, is contradicted by non-perturbative computation [46], and the second mechanism, reflection of baryon number from expanding bubble walls, according to naive estimates cannot provide a large enough asymmetry. 
number violation after the transition requires a large jump in the Higgs vev $v$ during the transition, i.e.

$$
v(T) / T \gtrsim 1[45] \text {. }
$$

Requiring that the baryon asymmetry of the universe be generated during the weak transition can give us new information about the $C P$ violating and Higgs sectors of the weak interactions, allowing us to rule out some models (such as the minimal standard model), and to constrain others. There is still very little known about the origin of $C P$ violation or weak symmetry breaking, and any information we can extract from the early universe is welcome.

\section{HIGH TEMPERATURE ELECTROWEAK BARYON VIOLATION}

\subsection{Baryon Violation and the Anomaly}

Classically conserved global $U(1)$ charges are well known to be potentially violated in quantum theories with fermions coupled to gauge fields; this can be seen through the one loop computation of the divergence of the corresponding current which leads to the anomaly. In the standard model the global baryon and lepton number currents are exactly conserved at the classical level. However the anomaly equations give:

$$
\partial_{\mu} J_{B}^{\mu}=\partial_{\mu} J_{L}^{\mu}=N_{f}\left(\frac{g^{2}}{32 \pi^{2}} W \tilde{W}-\frac{g^{\prime 2}}{32 \pi^{2}} X \tilde{X}\right)
$$

Here $N_{f}$ is the number of families, $W_{\mu \nu}$ is the $S U(2)$ field strength, $X_{\mu \nu}$ the $U_{Y}(1)$ field strength, and $g$ and $g^{\prime}$ are the associated gauge couplings. Note that the difference $B-L$ between the two charges is strictly conserved. These equations imply that fluctuations in the weak field-strength which have a non-zero dot product of the electric and magnetic fields will lead to corresponding fluctuations in baryon and lepton number. The right hand side of each of these equations is a total divergence: $W \tilde{W}=\partial_{\mu} K^{\mu}, X \tilde{X}=\partial_{\mu} k^{\mu}$ where

$$
\begin{aligned}
K^{\mu} & =\epsilon^{\mu \nu \alpha \beta}\left(W_{\nu \alpha}^{a} W_{\beta}^{a}-\frac{1}{3} g \epsilon_{a b c} W_{\nu}^{a} W_{\alpha}^{b} W_{\beta}^{c}\right) \\
k^{\mu} & =\epsilon^{\mu \nu \alpha \beta} X_{\nu \alpha} X_{\beta}
\end{aligned}
$$

and $W, X$ are the gauge potentials associated with the $S U(2)$ and $U_{Y}(1)$ groups respectively. Naively this would seem to allow the definition of a new baryon current which 
is exactly conserved, $J_{B}^{\mu}-N_{f} g^{2} / 32 \pi^{2} K^{\mu}+N_{f} g^{\prime 2} / 32 \pi^{2} k^{\mu}$. However $K^{\mu}$ is not gaugeinvariant, and this redefinition is not appropriate [57].

If we consider the change in the total baryon number from some initial time zero, to some final time $\tau$ (we will always take space to be a three-sphere, $S^{3}$ ) we can use the anomaly to give the change in the baryon number:

$$
\begin{aligned}
\Delta B & =N_{f}\left[N_{C S}(\tau)-N_{C S}(0)\right]-N_{f}\left[n_{C S}(\tau)-n_{C S}(0)\right] \\
N_{C S} & =\frac{g^{2}}{32 \pi^{2}} \int d^{3} x \epsilon^{i j k}\left(W_{i j}^{a} W_{k}^{a}-\frac{1}{3} g \epsilon_{a b c} W_{i}^{a} W_{j}^{b} W_{k}^{c}\right) \\
n_{C S} & =\frac{g^{\prime 2}}{32 \pi^{2}} \int d^{3} x \epsilon^{i j k} X_{i j} X_{k}
\end{aligned}
$$

We have written the result in terms of gauge non-invariant objects $N_{C S}$ and $n_{C S}$, but the difference at different times of each of these two objects is gauge invariant. We are generically interested in cases where initial and final average values of the gauge fieldstrengths are zero, and we wish to know if $\Delta B$ can be non-zero. In this case $n_{C S}$ is strictly zero, since it is proportional to the $U_{Y}(1)$ field strength, which is zero by assumption; hence from now on we need only deal with the $S U(2)$ gauge fields and $N_{C S}$. To compute $\Delta B$ we work in temporal gauge, $W_{0}=0$. Any gauge potential for which the field strength vanishes must then be a time independent gauge transformation of $\vec{W}=0$, and so we can choose our gauge such that $\vec{W}=N_{C S}=0$ at $t=0$. Then the potential at $t=\tau$ must be a (time-independent) gauge transformation of zero:

$$
\vec{W}(\tau)=h \vec{\nabla} h^{-1}
$$

In terms of $h$ we can write the change in baryon number as

$$
\Delta B=N_{f} N_{C S}(\tau)=N_{f} \frac{g^{2}}{32 \pi^{2}} \int d^{3} x \epsilon^{i j k} \operatorname{Tr}\left[h \partial_{i} h^{-1} h \partial_{j} h^{-1} h \partial_{k} h^{-1}\right]
$$

The problem of finding the change in baryon number has been reduced to the classification of the possible functions $h$, which are maps from $S^{3}$ into the gauge group $S U(2)$. The parameter $N_{C S}$ in this equation is a topological invariant of these maps, known as the Chern-Simons number. Thus the baryon number can change if the Chern-Simons number can be non-zero. As is well known, the possible values of the Chern-Simons number for $S U(2)$ are the integers, and so the baryon number can change by an integral multiple of the number of families. 


\subsection{Baryon Violation at Zero Temperature}

Although we have seen that the baryon number can change we have yet to see that it does, and we must still compute the rate for baryon violating processes. This requires an understanding of the dynamics of the fields as they go from the initial to final field configurations. It is this part of the problem which will require non-perturbative methods.

Non-perturbative effects in gauge theories began to receive attention in the seventies, especially in regard to the famous $U_{A}(1)$ problem, and the problem of baryon violation is not too different from the problem of axial charge violation in QCD [11]. Since the $U_{Y}(1)$ part of the anomaly does not play a significant role, for simplicity we will consider a pure $S U(2)$ gauge theory, without the gauged hypercharge. We will also restrict our attention to a single family - multiple families will then be easily dealt with.

If the standard gauge theory of weak interactions does violate baryon number by integral units we would expect to find a non-zero amplitude involving three quarks combined in a color singlet, plus one electron (or electron neutrino, depending on the electric charge of the three quarks). For example we can look at the matrix element $\langle u u d e\rangle$; a non-zero value for this object would be a sign of baryon violation. In the semi-classical approximation this matrix element is given by a path integral dominated by a stationary point, the weak instanton. The matrix element above is then evaluated by replacing the fields by their values at this stationary point, and multiplying by the determinant given by the Gaussian fluctuations about the instanton. So far, everything is identical to the QCD case. However, the weak interaction $S U(2)$ symmetry is spontaneously broken, and thus the instanton is not an exact stationary point; the Euclidean action coming from the Higgs potential can be reduced by shrinking the instanton radius to zero. However, these configurations are approximate stationary points, and should still be the dominant contribution to the functional integral. The integral over instanton sizes (including the action from the Higgs potential) can be performed to yield a non-zero matrix element which is exponentially small in the weak fine structure constant $\alpha_{w}$ as is characteristic of any semi-classical

process: $\langle u u d e\rangle \propto e^{-2 \pi / \alpha_{w}}$. The smallness of $\alpha_{w}$ implies that such a matrix element is totally unobservable - baryon violation in the form of low energy scattering experiments involving small numbers of particles is too small to be observed from this source.

The generalization to three families is, as promised, trivial. Since each left handed doublet has a zero mode in the instanton background, the only non-vanishing baryon violating matrix elements must involve at least one fermion from each doublet. Thus the change in baryon number during any anomalous event is three times what it would be for only one family. 


\subsection{Baryon Violation at non-Zero Temperature}

The semi-classical picture painted in the previous section can be thought of as a quantum tunneling: the instanton represents a barrier penetration, and the exponential in the coupling is similar to the conventional WKB factor in quantum mechanics. This picture can be made precise, by using a canonical Hamiltonian formulation of the gauge theory. The barrier penetration factor is then an integral along a path in configuration space of the usual WKB functional, which depends on the potential energy of the theory.

We can get an idea of how this "potential" varies over configuration space by finding its minima. We will first consider only the potential energy of the gauge field. One minimum is then easy to find: $W_{\mu}=0$ and no fermions, which we will call $\Omega_{0}$. We can conventionally take the value of this potential at $\Omega_{0}$ to be zero. Other potential minima have gauge fields which are of the form $W_{\mu}=h \partial_{\mu} h^{-1}$. At first sight these are not new minima - they are merely gauge transformations of $\Omega_{0}$. However if we consider a path in configuration space which begins at $\Omega_{0}$ and ends with $W_{\mu}=h \partial_{\mu} h^{-1}$, we know from the anomaly that the final configuration can have non-zero baryon and lepton number, both given by the Chern-Simons number of the gauge transformation $h$. Assuming that $h$ does have non-zero Chern-Simons number this configuration is then also a minimum of our potential with zero potential energy, but since it has non-zero baryon and lepton number is a genuinely different point in configuration space. Consequently the potential has an infinite number of minima, which we can label as $\Omega_{n}$, where $n$ is the change in ChernSimons number relative to $\Omega_{0}$ (a gauge invariant quantity); note that this is also just the baryon number per family. All that remains is to find the potential at all other points of the infinite-dimensional configuration space - an impossible task. However we can plot a one-dimensional path in this space which goes through all of these minima, and we choose the path for which the height of the barrier between minima is as small as possible. The resulting one-dimensional potential is given in fig. 1.

The field configuration at the top of the barrier is called the "sphaleron" [13], which has potential energy:

$$
E_{\mathrm{sp}}=\frac{2 M_{W}}{\alpha_{w}} B\left(\lambda / g^{2}\right)
$$

where $B$ is a constant requiring numerical evaluation; for the standard model with a single Higgs doublet this parameter ranges between $1.5 \leq B \leq 2.7$ for $\lambda / g^{2}$ varying between 0 and $\infty$ ( $\lambda$ is the Higgs self coupling). 
Although quantum tunneling through this barrier is irrelevant due to the small coupling, the finite (although large) barrier height can have important consequences for baryon violation. So far we have assumed that the initial and final configurations in our baryon violating transitions have no gauge field, but only an assortment of low energy fermions with baryon number differing by one; this is the case for the S-matrix element $\langle u u d e\rangle$. But we might also consider processes that have more complicated initial and final configurations, with a total energy greater than the barrier height. In this case quantum tunneling is not necessary, as we have enough energy present in the initial configuration to cross the barrier. This is the essence of the finite temperature baryon violation argued by Kuzmin et al. 15] - at temperatures near the sphaleron energy initial configurations with energies above the barrier will be likely members of the thermal ensemble, this likelihood being given by a Boltzmann factor, and baryon number changing transitions can occur without barrier penetration. At high temperature baryon number changes by diffusion across the top of the barrier, with a rate proportional to $\exp \left(-E_{\mathrm{sp}} / T\right)$.

We can gain some insight into this problem by imagining a one-dimensional particle analogue, a pendulum in the gravitational potential of the earth. This pendulum will be the analog of the gauge field (or at least one degree of freedom of the gauge field). For the baryons, we imagine that each time the pendulum passes through an angle of $\pi$ (the point where the pendulum points straight up) traveling in the clockwise direction it moves a lever which increases a counter by one; when traveling through $\pi$ in the counterclockwise direction the motion of the lever decreases the counter by one. The configuration space of this example consists of the angle of the pendulum (between 0 and $2 \pi$ ) and the value of the counter $(B)$, and the classical potential energy of the pendulum is precisely our fig. 1. At zero temperature, barrier penetration will occur, allowing transitions among configurations with different values of the counter; if the barrier is high, these processes are suppressed, as are the tunneling events in our gauge theory. At finite temperature however, the pendulum will experience thermal fluctuations, and at sufficiently high temperature will frequently cross $\theta=\pi$, randomly in both directions. Since each time the pendulum crosses $\pi$ the counter value changes, we see the value of $B$ fluctuate. If we were to start the system with the counter localized at some point in configuration space then $B$ will diffuse away from this value like $\sqrt{t}$, following a typical random walk.

While this picture does show fluctuations in the counter (and by analogy local fluctuations in baryon number in the gauge theory case), the average value of the counter does not change since fluctuations in each direction are equally likely; this is not yet the 
physics of baryon violation that we are looking for. As emphasized by Kuzmin et al. [15], the average value of the baryon number can change only if there is some bias which favors fluctuations in one direction over those in the other. The potential of fig. 1 does not show any such bias; fortunately this is the result of our leaving out an essential part of the physics. We have so far plotted only the potential associated with the gauge field, but there is of course an energy associated with the baryons as well. For a sufficiently dilute system this is just the energy of a free Fermi gas with fixed baryon and lepton number, while at high density it is more complicated. In either case its minimum will be at zero baryon and lepton number, and will be symmetric about this value. In our analogy we can associate a quadratic potential with the value of the counter, with a minimum at zero. Thus there is some curvature associated with the periodic potential, which we show in the upper curve of fig. 1. This additional effect provides the bias we are looking for; if we start the system localized near some value of the baryon density, it diffuses outwards eventually reaching an equilibrium with zero average baryon number. The bias of the rate in the direction of increasing baryon number relative to the decreasing direction is given by detailed balance - assuming that this bias is small we may write [20]:

$$
\frac{d n_{B}}{d t}=3\left(\Gamma_{+}-\Gamma_{-}\right)=-3 \frac{\Gamma_{a}}{T} \Delta F
$$

where $\Delta F$ is the free energy difference between neighboring minima, and $\Gamma_{a}$ is the rate per unit volume for fluctuations between neighboring minima in the absence of bias (i.e. in the absence of fermions). We have assumed that the processes which equilibrate all degrees of freedom aside from the baryon number (and the corresponding degree of freedom of the gauge field) are rapid compared to the diffusion time, and this is why the free energy appears rather than the internal energy.

\subsection{Rates}

The first serious attempt to compute the anomalous rate $\Gamma_{a}$ used a technique developed by Langer [58] and Affleck [59] for evaluating the diffusion rate of a system over a barrier at finite temperature. The procedure effectively reduces the theory to one dimension (the degree of freedom we plotted above), computes the flux of the system across the barrier in one direction weighted with a Boltzmann factor for each possible initial state, and finally reintroduces the degrees of freedom transverse to this one mode in a quadratic approximation with a thermal occupation. The idea behind this technique is to treat the 
most important mode, the one which goes over the barrier most easily, as exactly as possible, and then use only the small fluctuations around this mode. The resulting computation should be valid when the rate of thermal diffusion across the barrier is small, and the trajectory over the sphaleron barrier is the most important. The result has all the features that we have described so far, especially the characteristic Boltzmann factor associated with the sphaleron energy [16, 19]:

$$
\Gamma_{a}=\gamma\left(\alpha_{w} T\right)^{-3} M_{W}^{7} e^{-E_{\mathrm{sp}} / T} \quad \text { (broken phase) }
$$

Relatively simple arguments have been given for this general form including the exponential and the $M_{W}^{7}$ dependence [17, 20]. The constant $\gamma$ cannot be calculated analytically; it depends on the ratio of the Higgs self-coupling to the gauge coupling squared and has been numerically evaluated in ref. [19]. From this form we can see that the Boltzmann factor is, naively, a significant suppression up to temperatures of hundreds of GeV. Note however that the energy of the sphaleron configuration is proportional to the weak symmetry breaking - if the weak interactions were unbroken, we could find a path in configuration space which changes the baryon number by one unit, with arbitrarily small potential energy at each point. Although the energy in this case is arbitrarily small, the integral of the WKB factor along this path is still bounded below by $8 \pi^{2} / g^{2}$, and consequently quantum tunneling is suppressed for any value of the symmetry breaking. On the other hand, the thermodynamic baryon violation does not care about the tunneling factor, but only about the energy of the configuration, and consequently in the symmetric phase we can change the baryon number with neither suppression from quantum tunneling, nor suppression from a small Boltzmann factor. This means that baryon violation is only suppressed up to temperatures where the weak interactions undergo a transition to the symmetric phase, which occurs at a temperature around one hundred GeV. Thus we need the rate of baryon violation not where the Boltzmann factor is a large suppression and the calculation of [19] is applicable, but also in the symmetric phase where there is no Boltzmann suppression factor at all!

The calculation of the baryon violation rate in the symmetric phase is considerably more difficult than in the broken phase at low temperatures. The problem is that infrared divergences begin to invalidate a perturbative expansion when the temperature is higher than all mass scales of the theory. These divergences are actually cut off by the generation of a "magnetic screening length" $\xi_{M}$ which gives the scale of spatial correlations in 
the gauge theory at high temperature, but no reliable perturbative scheme involving this screening exists [60, 61]. A simple scaling argument allows us to estimate $\xi_{M}$. At high temperature spatial correlations become increasingly classical, and we need only consider the classical dynamics of the gauge field. But in this case the Hamiltonian appears only in the combination $H / T$. Classically the gauge coupling is an irrelevant parameter; by rescaling the fields we can always bring the coupling out as a factor $1 / \alpha_{w}$ in front of the Hamiltonian. Thus the only place the coupling appears is in the combination $\alpha_{w} T$, and therefore on dimensional grounds the screening length is $\xi_{M} \sim\left(\alpha_{w} T\right)^{-1}$. Similarly we can estimate the rate per unit volume of baryon violating transitions as

$$
\Gamma_{a}=\kappa\left(\alpha_{w} T\right)^{4} \quad \text { (symmetric phase) }
$$

where $\kappa$ is a dimensionless constant which requires something beyond a simple scaling argument to compute. Ambjorn et al. [18] have attempted to evaluate this rate numerically, and find a value $0.1 \lesssim \kappa \lesssim 1.0$. These computations are difficult, suffering from both infrared and ultraviolet divergences; the results are noisy, and the diffusive behavior that one expects is not obvious from the data (for example, growth of the fluctuations in the Chern-Simons number as $\sqrt{t}$ cannot be verified). For these reasons the results must be taken as only indicative of the true value.

Recognizing the difficulties of simulating the standard model in $3+1$ dimensions, Grigoriev, Rubakov \& Shaposhnikov [62 have instead simulated the classical equations of motion for a $1+1$ dimensional analog of the standard model, the Abelian Higgs model. This theory has an anomalous fermion number, instantons, and sphalerons similar to the $3+1$ dimensional gauge theory, but is both easier to simulate, and has better ultraviolet behavior than its four dimensional analog. Grigoriev et al. used a Metropolis algorithm to generate an initial field configuration; since the theory is non-linear and contains an infinite number of degrees of freedom, its behavior is hoped to be ergodic, and this initial configuration should give "thermal" results, with a temperature associated with the statistical ensemble from which it is picked. The classical equations of motion were then used to evolve this configuration forward in time. The result of this evolution shows striking confirmation of the thermal diffusion across the barrier; as fig. 2 shows, the system spends most of its time near one (integer) value of the Chern-Simons number, with only small oscillations around this value. However occasionally the system makes a rapid transition to a neighboring integer value of $N_{C S}$, and then continues to hover around this new value. The rate of 
these transitions is in good agreement with the $1+1$ dimensional analog of (2.8), showing the Boltzmann factor associated with diffusion over a barrier. In addition, the rate and amplitude of the small fluctuations around one of these integral values is measured to be in agreement with analytic estimates. Grigoriev et al. looked in detail at the field configurations in the neighborhood of these rapid transitions and were able to verify that the field indeed "unwinds" by passing over the barrier between neighboring minima of the potential as we have discussed.

\subsection{Baryon Violation at the Phase Transition}

There are several issues which become relevant specifically for baryon violation at the electroweak phase transition. If this phase transition is first order and proceeds through the nucleation of bubbles of true vacuum with non-zero symmetry breaking amidst a sea of false vacuum, we will then need to know the rate of baryon violation not only in the symmetric phase (given by $(2.9))$ or the broken phase when $\exp \left(-E_{\mathrm{sp}} / T\right)$ is small (given

by $(2.8)$ ) but also near the interface of the two phases, the bubble wall. In this case we need to know how the baryon violation rate makes a transition between these two forms. A naive interpolation between these two formulæ as is done in ref. [63] would indicate that the region of the bubble wall where baryon violation is significant would be the region where

$$
M_{W}(T, \phi) \lesssim 7 \alpha_{w} T \quad \text { (condition for rapid baryon violation) }
$$

Here $M_{W}(T, \phi)$ is the value of the $W$ boson mass which is varying through the wall. The rate in this region is still not well known but is likely to be similar to the rate outside the bubble. These statements are not definitive since the only known explicit computational methods used in ref. [19] are not reliable at this point.

An entirely different problem has been raised by Turok et al. [64, 65]. As the bubble walls created at the weak phase transition expand, they liberate energy in the conversion of the false vacuum to the true vacuum. This liberated energy may move the system away from thermal equilibrium in the broken phase behind the bubble wall. Then, as is suggested by some simulations in $1+1$ dimensions, none of the formulas above are directly relevant, since they assume that most of the degrees of freedom of the system are in equilibrium. Turok et al. find that significant Chern-Simons fluctuations persist in this broken phase region, contrary to the thermal rate predicted from ref. (2.8). They find that the rate for these fluctuations actually increases as the temperature is lowered. However Dine has 
argued qualitatively that this phenomenon does not occur in the real $3+1$ dimensional world [66]. At the weak transition the available latent heat is insufficient to create a field configuration localized near the top of the sphaleron barrier - the latent heat in a sphaleron volume is $\sim\left(\pi \lambda / g^{3}\right) T$, while the corresponding height of the barrier is $\sim(4 \pi / g) T$, and for a transition in which eq. (1.1) is satisfied $\lambda \lesssim g^{3}$. This analysis disagrees with ref. [64] where the relevant volume has been taken to be the magnetic screening volume $\left(\xi_{M}\right)^{3}$ rather than the smaller sphaleron volume. This interesting possibility will have to be pursued by numerical investigations in $3+1$ dimensions.

\section{THE WEAK PHASE TRANSITION}

\subsection{The Nature of the Transition}

The nature of the weak phase transition in the minimal standard model is fairly well understood in two different limits. The transition is known to be strongly first order in the Coleman-Weinberg scenario [67-71, where the Higgs mass is tuned so that at zero temperature the curvature of the effective potential for the Higgs vanishes at the origin. By "effective potential", we mean the free energy density for a spatially homogeneous Higgs field as computed in perturbation theory by the methods of Coleman \& Weinberg 67, 72]. For values of the Higgs field between two local minima this is not the "true" effective potential, which includes spatially inhomogeneous field configurations and is always convex [73, 74. The Coleman Weinberg effective potential in principle is relevant for computing properties of the phase transition such as the existence of metastable phases and bubble nucleation rates [58, 72]. In the minimal standard model the Coleman Weinberg scenario requires a Higgs mass of $10 \mathrm{GeV}$, which is ruled out; however in models with several scalars this scenario is still viable [75, 76]. As the mass of the Higgs increases, the strength of the transition decreases. Perturbative calculations [45, 68, 71, 78,88] indicate a first order transition persists as the Higgs mass increases. However in the minimal standard model the lower bound on the Higgs mass is currently $60 \mathrm{GeV}$, comparable to the $\mathrm{W}$ mass 89 . For Higgs masses greater than the gauge boson masses finite temperature perturbation theory is unreliable at the critical temperature [41, 83, 88, 90,93]. In the limit where the Higgs mass is much heavier than the $\mathrm{W}$ and $\mathrm{Z}$ masses, it is a reasonable approximation to neglect the gauge and Yukawa interactions when studying the transition. Then the minimal standard model is approximately the linear sigma model for $\mathrm{O}(4)$ symmetry breaking. The finite temperature phase transition for the $\mathrm{O}(4)$ model has been studied 
numerically using lattice field theory [71, 94, 95] and by imposing an infrared cutoff which is removed using the renormalization group [96], and is clearly second order. Thus for a heavy Higgs the transition is at most very weakly first order, as is also verified by lattice calculations including the gauge couplings [97]. Computations with $\mathrm{N}$ flavors of scalars in the large $\mathrm{N}$ limit confirm the result that the transition is second order or weakly first order for a heavy Higgs, and first order for a sufficiently light Higgs [77, 98, 99]. It has also been argued using the renormalization group (see ref. [100]) that the transition is first order for small Higgs mass and second order for large Higgs mass [101.

The weak phase transition is under active investigation since demonstrating the feasability of electroweak baryogenesis and doing a precise computation of the resulting baryon abundance requires an understanding of the details of this transition - the order, the critical temperature, the bubble nucleation rate, the velocity and shape of the expanding walls, and the Higgs vev after the transition. Most of these details depend on the model of the weak symmetry breaking sector, about which we currently have very little experimental information. Requiring a first order transition with a sufficiently large Higgs vev after the transition places significant constraints on this sector. For instance, in the minimal standard model the condition (1.1) for avoiding washout of the baryon number after the transition places an upper bound on the Higgs mass [45]. The numerical value of this bound is still uncertain, but perturbative calculations place it in the experimentally ruled out $30-45 \mathrm{GeV}$ range [45, 80, 84, 63, 102, 103]. In models with additional scalars there is a weaker upper bound on the mass of the lightest scalar [76, 81, 104 110]. For instance Anderson \& Hall [81] showed that simply adding a gauge singlet scalar $s$ with a $s^{2}|H|^{2}$ coupling substantially weakens the Higgs mass bound. Similarly, Myint [106] showed that in the minimal supersymmetric standard model, the coupling of the top squarks to the Higgs doublet weakens the Higgs mass bound slightly.

The main theoretical tools for studying the transition are finite temperature perturbation theory and lattice field theory. The lattice studies of the finite temperature transition for most of the interesting cases, such as for the minimal standard model including gauge boson effects with a relatively light Higgs, are still in a preliminary stage 171, 97, 111, 112], and do not contradict improved one loop perturbation theory [77, 78, 83, 86, 113 [15]. Computing the effective potential for the order parameter $\phi$ gives the order of the transition - if at the critical temperature there are two degenerate minima the transition is first order. In fig. 通 we plot the effective potential at various temperatures for a typical example of a first order phase transition. 
The perturbative one loop result for the effective potential $V$ is [67, 68, 77, 78]

$$
\begin{aligned}
V(\phi, T)= & V_{\mathrm{cl}}(\phi)+\left(\frac{1}{64 \pi^{2}}\right) \sum_{i}(-1)^{2 s_{i}} M_{i}^{4}(\phi) \ln M_{i}^{2}(\phi)+(\text { polynomial in } \phi) \\
& +\sum_{i}(-1)^{2 s_{i}} T \int \frac{k^{2} d k}{\left(2 \pi^{2}\right)} \ln \left[1-(-1)^{2 s_{i}} \exp \left(-\frac{\sqrt{k^{2}+M_{i}^{2}(\phi)}}{T}\right)\right],
\end{aligned}
$$

where $M_{i}$ and $s_{i}$ are the mass and spin respectively of the $i$ 'th particle, the sum on $i$ includes all degrees of freedom including spin, $V_{\mathrm{cl}}$ is the classical potential and the polynomial in $\phi$ is determined by the choice of renormalization condition. Physically this result is interpreted as the ground state energy plus the free energy of a gas of noninteracting particles at finite temperature. We therefore do not expect eq. (3.1) to be accurate at arbitarily high temperatures, where the density of thermal particles is too high to neglect interactions. Examination of higher order corrections for a theory with gauge interactions and scalar self coupling $\lambda$ shows that the naive finite temperature perturbation theory at high temperature is an expansion in powers of $\lambda T^{2} / M_{\phi}^{2}(\phi)$ and $g_{i}^{2} T^{2} / M_{i}^{2}(\phi)$, where $g_{i}$ is the coupling of the $i$ 'th gauge particle with mass $M_{i}$ to $\phi$. Clearly this expansion is not valid at high temperatures, or for any values of $\phi$ for which there are massless interacting particles. However this one loop result can be improved at high temperatures, by summing up the dominant high temperature corrections to all orders. The leading corrections, which grow as $T^{2}$, come from subgraphs with superficial degree of divergence $2-i$.e. corrections to the boson propagators [77, 78]. How best to improve perturbation theory is currently a subject of active investigation [83 [86, 113-[119]. Here we will summarize the basic idea of modifying the boson propagators to sum a class of diagrams which give the leading high temperature corrections. For $T$ much larger than $M_{i}$ the integral in eq. (3.1) becomes approximately

$$
V_{1}^{\text {boson }} \approx \frac{M_{i}^{2}(\phi) T^{2}}{24}+\text { const }+\mathcal{O}(\mathrm{T})
$$

for a boson and

$$
V_{1}^{\text {fermion }} \approx \frac{M_{i}^{2}(\phi) T^{2}}{48}+\text { const }+\mathcal{O}(1)
$$

for a fermion, and gives an effective temperature dependent contribution to the scalar mass

$$
\begin{aligned}
M_{\phi_{\mathrm{eff}}}^{2} & =V_{\mathrm{cl}}(\phi)^{\prime \prime}+\Delta M_{\phi_{\mathrm{eff}}}^{2} \\
\Delta M_{\phi_{\mathrm{eff}}}^{2} & =\sum_{i}\left[\frac{\left(3+(-1)^{2 s_{i}}\right)}{96}\right] M_{i}^{2}(\phi)^{\prime \prime} T^{2} .
\end{aligned}
$$


Including this mass in the scalar propagator is equivalent at one loop to the summation of all the leading high temperature contributions of the "daisy" graphs such as those in fig. $4[77,78]$. In perturbation theory there is also a temperature dependent contribution to the effective mass of the longitudinal gauge bosons - the usual Debye mass of order $g T$. The transverse components of the gauge bosons are believed to get a non perturbative contribution to their masses of order $\alpha_{w} T$, but remain massless to all orders in perturbation theory. A systematic way of including the leading $\mathcal{O}\left(T^{2}\right)$ high temperature contribution to the $\phi$ propagator 87, 115] is to add and subtract from the Lagrangian a term

$$
\mathcal{L} \rightarrow \mathcal{L}+\frac{\phi^{2}}{2} \Delta M_{\phi_{\mathrm{eff}}}^{2}-\frac{\phi^{2}}{2} \Delta M_{\phi_{\mathrm{eff}}}^{2}
$$

where the first term is treated as part of the unperturbed Lagrangian and included in the scalar propagator while the second term is treated in perturbation theory as an interaction which cancels the leading high temperature pieces of thermal subloops. A similar but more complicated method can be used to sum the leading high temperature contribution to the gauge boson propagators (see ref. [87]). Improved perturbation theory then becomes an expansion in powers of $\lambda T / M_{\phi}(\phi)_{\text {eff }}$ and $g_{i}^{2} T / M_{i}(\phi)_{\text {eff }}$, up to logarithms. Because the effective thermal masses of the particles grow linearly with $T$ (for large $T$ ) the improved expansion does not necessarily break down at high temperature. It always breaks down near a second order phase transition however, since $M_{\phi}(0)$ eff goes to zero as the critical temperature is approached, and is also unreliable in the vicinity of a very weakly first order transition. For a sufficiently strongly first order transition, it is possible for the expansion parameters to remain small in both minima of the effective potential at the critical temperature.

For the minimal standard model with a Higgs mass in the (30-80) GeV range, a decent approximation to the improved one loop effective potential is to compute eq. (3.1) using the temperature dependent masses for the bosons and a high temperature approximation for the integrals, the result is [84]:

$$
V(\phi, T) \approx \frac{\lambda_{T}}{4} \phi^{4}-\frac{\left(2 m_{W}^{3}+m_{Z}^{3}\right) T}{6 \pi v_{0}^{3}}|\phi|^{3}+\frac{\left(2 m_{W}^{2}+m_{Z}^{2}+2 m_{t}^{2}\right)\left(T^{2}-T_{0}^{2}\right)}{8 v_{0}^{2}} \phi^{2}+\text { const }
$$

where

$$
\begin{aligned}
T_{0}^{2} & =\frac{2 v_{0}^{2}\left[m_{H}^{2}-3\left(2 m_{W}^{4}+m_{Z}^{4}-4 m_{t}^{4}\right) /\left(8 \pi^{2} v_{0}^{2}\right)\right]}{\left(2 m_{W}^{2}+m_{Z}^{2}+2 m_{t}^{2}\right)}, \\
\lambda_{T} & =\lambda-\frac{3}{16 \pi^{2} v_{o}^{4}}\left(2 m_{W}^{4} \ln \frac{m_{W}^{2}}{a_{B} T^{2}}+m_{Z}^{4} \ln \frac{m_{Z}^{2}}{a_{B} T^{2}}-4 m_{t}^{4} \ln \frac{m_{t}^{2}}{a_{F} T^{2}}\right), \\
\ln a_{B} & =2 \ln 4 \pi-2 \gamma \approx 3.91, \\
\ln a_{F} & =2 \ln \pi-2 \gamma \approx 1.14 .
\end{aligned}
$$


Note the presence of the $|\phi|^{3}$ term, which means that at the critical temperature there will be two coexisting minima, hence a first order phase transition [68]. For the range of Higgs masses considered, this critical temperature $T_{c}$ will be just above $T_{0}$. Is this calculation reliable? At the critical temperature, the effective potential has the form

$$
V\left(\phi, T_{c}\right) \approx \frac{\lambda_{T_{c}}}{4}\left[\phi-\frac{\left(2 m_{W}^{3}+m_{Z}^{3}\right) T_{c}}{3 \pi v_{0}^{3} \lambda_{T_{c}}}\right]^{2} \phi^{2} .
$$

Note that the condition (1.1) requires

$$
\lambda_{T_{c}} \lesssim g^{3}
$$

The effective scalar mass in each of the two minima is

$$
M_{\phi_{\mathrm{eff}}} \approx \sqrt{\frac{1}{2 \lambda_{T}}} \frac{\left(2 m_{W}^{3}+m_{Z}^{3}\right) T_{c}}{3 \pi v_{0}^{3}} .
$$

In either minimum, additional loops involving the scalar self coupling will be suppressed by

$$
\sim \frac{\lambda_{T_{c}} T_{c}}{M_{\phi_{\mathrm{eff}}}}=\frac{\sqrt{2 \lambda_{T_{c}}^{3}} 3 \pi v_{0}^{3}}{\left(2 m_{W}^{3}+m_{Z}^{3}\right)} \sim \frac{m_{H}^{3}}{\left(2 m_{W}^{3}+m_{Z}^{3}\right)} .
$$

The gauge bosons in the broken phase and the longitudinal gauge bosons in the symmetric phase are heavier than the scalars and will give rise to corrections proportional to powers of $g$. However the transverse gauge boson masses in the symmetric phase vanish in perturbation theory; perturbative corrections involving these states become unsuppressed for $\phi<g T$ and are infrared divergent, making a perturbative calculation of the effective potential at the origin impossible [60, 68, 61]. If we assume that these modes receive a nonperturbative mass of order $\alpha_{w} T$ (related to the magnetic screening length), the uncertainty in the value of the effective potential at the origin is $\mathcal{O}\left(g^{6} T^{4}\right)$ due to nonperturbative effects [60, 61]. Nevertheless it is still possible to establish the order of the transition in perturbation theory. If $\lambda_{T_{c}}$ is small (light Higgs mass) when compared with $g^{2}$ then examination of eq. (3.8) shows that the barrier between the two minima occurs at relatively large $\phi$ and the height of the barrier is larger than the $g^{6} T^{4}$ uncertainty in the value of the effective potential in the symmetric phase. (The calculation of the effective potential also breaks down near the inflection points, but a lower bound on the barrier height can be inferred from the value of the potential in the region between the minimum and the inflection 
point where the perturbation expansion parameters are small.) Thus while the nonperturbative effects will induce some uncertainty into the value of the critical temperature, they will not affect the result that the transition is first order provided that

$$
g T_{c} \ll \frac{\left(2 m_{W}^{3}+m_{Z}^{3}\right) T_{c}}{3 \pi v_{0}^{3} \lambda_{T_{c}}},
$$

which implies that $m_{H}<m_{W}$. In the minimal standard model with a Higgs mass in the experimentally allowed range eq. (3.12) is at best marginally satisfied, and so perturbative calculations should not be terribly accurate. More sophisticated calculations validate this conclusion-for instance Arnold \& Espinosa [87 and Bagnasco \& Dine 88] find fairly large $(40 \%)$ two loop corrections to the improved one loop result for the value of the Higgs vev at the critical temperature in the minimal standard model with a $60 \mathrm{GeV}$ Higgs. Clearly better methods are needed for quantitative results, such as nonperturbative lattice calculations.

\subsection{Dynamics of the Transition in the Early Universe}

The conventional picture of how a first order cosmological transition proceeds is that as the universe expands it cools in the symmetric phase until it reaches the critical temperature $T_{c}$ at which time the broken phase becomes equally favored. At $T \simeq T_{c}$ bubbles of the broken phase nucleate, but surface tension effects cause these to immediately shrink, so that the universe remains in an approximately homogeneous state. For $T$ somewhat below $T_{c}$, bubbles of broken phase nucleate which are sufficiently large so that the volume pressure from the lower free energy inside the bubble can overcome the surface tension. These critical bubbles grow until the universe is completely converted to the broken phase. The time scale for the formation of a critical bubble is proportional to $\exp \left(F_{c} / T\right)$, where $F_{c}$ is its free energy. At $T=T_{c}, F_{c}=\infty$ and so the universe always supercools, resulting in a departure from thermal equilibrium [58]. A detailed study of the bubble growth in the minimal standard model is given in ref. [120].

This picture was recently challenged by several authors who claimed that thermal fluctuations in the order parameter could maintain thermal equilibrium even during a first order transition [90,92], which would make baryogenesis impossible. For instance subcritical bubbles of the broken phase could appear above the critical temperature, and during the transition the universe could be an emulsion of both phases with the fraction of broken phase gradually increasing. Such large thermal fluctations would also destroy the 
validity of perturbation theory, since perturbative calculations assume that the deviations from a homogeneous field configuration are small [84, 93, 121]. Fortunately, for those models in which eq. (1.1) is satisfied such thermal fluctuations are suppressed, perturbative expansion parameters are less than 1 , and the conventional picture is probably correct 84, 93, 121, 122.

Another conventional assumption is that the bubbles are nearly spherical and that, once they have grown to macrosopic size, it is a good approximation to take the walls to be planar. It has been suggested [123 that smooth walls are unstable towards developing wrinkles, which would greatly complicate bubble evolution, but a recent computation [124] indicates that for the weak transition smooth walls are stable and that surface tension will keep the expanding bubbles spherical until they collide.

\subsection{Propagation and Shape of the Bubbles}

In the conventional picture of the transition local departure from thermal equilibrium, and baryogenesis, primarily occurs in the vicinity of the expanding bubble walls. A quantitative calculation of the baryon number of the universe as described in the next section requires the temperature at which bubbles nucleate, the wall profile, and the wall velocity. If the effective action is known, computing the nucleation temperature and the wall profile is a straightforward exercise [12, 58, 59, 125]. The nucleation temperature is the temperature where the rate per unit volume of critical bubble nucleation equals the age of the universe multiplied by the volume inside the horizon. The critical bubble nucleation rate depends on $F_{c}$, which can be computed by finding a spherically symmetric field configuration $\phi(r)$ which is a stationary point of the effective action and has $\phi=0$ for large $r$, and $\phi$ near the minimum of the effective potential for small $r$. The free energy for such a configuration is approximately (neglecting corrections to the derivative terms)

$$
S[\phi(r)] \approx \int r^{2} d r\left[\frac{1}{2}\left(\phi^{\prime}\right)^{2}+V(\phi, T)\right]
$$

and a stationary point can be obtained by finding a nontrivial solution of

$$
\frac{1}{r^{2}} \frac{d}{d r}\left(r^{2} \phi^{\prime}\right)-\frac{\partial V}{\partial \phi}=0
$$

satisfying the boundary conditions

$$
\phi(\infty)=0, \quad \phi(0)^{\prime}=0
$$


This must be done numerically, although an approximate analytic formula is given in ref. [84]. In the minimal standard model with a Higgs mass in the $30-80 \mathrm{GeV}$ range the result is that the nucleation temperature is nearly the critical temperature [63, 81, 82, 84, 103. The wall profile for the boundary between the two coexisting phases at the critical temperature is the solution to the differential equation (3.14) at $T=T_{c}$ in the large $r$ limit, subject to the boundary conditions $\phi(\infty)=0$ and $\phi(-\infty)=v$, where $v$ is the vev in the broken phase. This solution should also give a good estimate for the profile of the wall of an expanding bubble. (The exact wall shape of an expanding bubble in a thermal medium is still unknown.) If the effective potential at the critical temperature is $(\lambda / 4)(\phi-v)^{2} \phi^{2}$, the analytic solution for a wall centered at $r=0$ is

$$
\phi=\frac{v}{2}\left[1+\tanh \left(-r v \sqrt{\frac{\lambda}{8}}\right)\right] .
$$

The width of the wall $\delta_{w} \equiv(\sqrt{8 / \lambda}) / v$ is quite model dependent, which is unfortunate since, as we show in $\S 4$, this is an important parameter for calculating the baryon number produced. In the minimal standard model, if one takes the Higgs mass to have the experimentally ruled out value $35 \mathrm{GeV}$ so that $v / T \approx 1$, the wall width 3 is $\delta_{w} \sim 24 / T$. In general $\delta_{w} \propto v / \sqrt{h}$, where $h$ is the barrier height between the two minima of the effective potential. In a model with a strongly first order transition, such as a version of the singlet majoron model with the potential tuned to have a nearly flat direction [76], or a supersymmetric model with a gauge singlet superfield [110], the wall can be thin, $\delta_{w}=\mathcal{O}(1 / T)$. These calculations require knowing the effective potential in the region between the two minima, and perturbation theory breaks down at the inflection points. In between the inflection points the perturbative potential has an imaginary part, arising from the classical instability of the homogeneous field configuration which we have assumed dominates the perturbative expansion [72]. However the lifetime of such a homogeneous configuration is, in most cases, long enough that it is meaningful to compute its free energy, which is approximately given by the real part of the effective potential [72]. Any smooth continuation of the effective potential from the regions where perturbation theory is sensible will not deviate too far from the perturbative value, and for a sufficiently strongly first order transition in a weakly coupled theory the perturbative calculations should give a reasonable estimate for nucleation rates and the wall width $\delta_{w}$.

3 This estimate differs from ref. 84 because of a factor of two difference in the definition of the wall width. 
The calculation of the wall velocity $v_{w}$ is more complicated, since it involves nonequilibrium interactions of the thermal plasma with the wall. The higher pressure inside the bubble will tend to accelerate the wall, and the scattering of thermal particles from the wall will dissipate energy and slow the wall down [125]. A plausible assumption is that the wall will reach a terminal velocity where the different forces balance. This assumption was challenged by Turok [126], who pointed out that if the temperature is spatially constant and the particle distributions are always the local thermal distributions for a given value of $\phi$, then the force on the wall is independent of velocity and continuously accelerates the wall. This is of course true, since the wall can only slow down by dissipating energy, which will cause a local departure from equilibrium. Thus if the wall is extremely thick when compared with particle mean free paths, so that the particle distributions approximately equilibrate with the changing value of $\phi, v_{w}$ will be ultrarelativistic. For a wall which is not infinitely thick, particle interactions with the moving wall will affect their distributions in a velocity dependent way. For instance weak gauge bosons and top quarks, which are massless in the symmetric phase and have large masses in the interior of the bubble, will tend to reflect from the wall. The reflected particles absorb some of the wall's momentum and the distribution of reflected particles is nonthermal. Several estimates of $v_{w}$, which include the effects of particle interactions with the wall and rethermalization in various approximations, indicate that $v_{w} \sim 0.1 c$ for a wall of width $\delta_{w} \simeq 1 / T$ and is at most mildly relativistic for $\delta_{w} \simeq(10-30) / T$ [63, 84, 127]. These calculations assume the minimal standard model phase transition parameters with only $\delta_{w}$ allowed to vary; in general $v_{w}$ is model dependent.

\section{CALCULATING THE BARYON ASYMMETRY}

The master equation for weak scale baryogenesis is eq. (2.7), which may be rewritten as

$$
\frac{d n_{B}}{d t}=-9 \frac{\Gamma_{a}}{T} \frac{\partial F}{\partial B} \equiv-9 \frac{\Gamma_{a} \mu_{B}}{T}
$$

where we have replaced $\Delta F=3 \partial F / \partial B$, the derivative computed at fixed $B-L$, remembering that $\Delta B=3$ in an anomalous event. The rate $\Gamma_{a}$ is given in eqs. (2.8), (2.9). We have given above the reasons to suspect that two of the three necessary ingredients for baryogenesis may exist at the weak phase transition: baryon violation and departure from thermal equilibrium. There remain the requirements of $C$ and $C P$ violation, as well as some concrete mechanism that explains how a nonzero $\mu_{B}$ can arise dynamically during the weak phase transition. 


\section{1. $C P$ violation}

We wish to explain the observed baryon to entropy ratio $n_{B} / s \simeq(0.6-1) \times 10^{-10}$ [1]. From the expression (2.9) for the anomalous rate $\Gamma_{a}$ we expect a factor of $\alpha_{w}^{4} \approx 10^{-6}$ in our final answer for $n_{B} / s$, and since $s=\left(2 \pi^{2} g_{*} / 45\right) T^{3}$ with $g_{*} \sim 10^{2}$ at the weak scale, one can only tolerate an additional suppression of order $10^{-2}$ from the combined effects of $C P$ violating angles $\delta_{C P}$, and various dynamical effects. This guarantees that $C P$ violating effects must be large at weak scale energies, further constraining possible models and giving reason to hope that new $C P$ violating effects may be experimentally accessible beyond those seen in the kaon system.

It is natural to wonder whether the requisite $C P$ violation at the weak scale could be of the Kobayashi-Maskawa (KM) form. At high temperature one may expect that that a dimensionless measure of $C P$ violation $\delta_{C P}$ in the standard model could only depend on a reparametrization invariant combination of the dimensionless Yukawa couplings and mixing angles. In $\S 1$ we argued that $\delta_{C P} \simeq 10^{-20}$, a suppression factor apparently ruling out KM $C P$ violation as playing any role in weak scale baryogenesis. In the kaon system, the $C P$ violating parameter $\epsilon$ is seventeen orders of magnitude larger than this estimate because the $C P$ conserving contribution to $\overline{K^{0}}-K^{0}$ mixing is so small. It is conceivable that dimensionless numbers such as $m_{p l} / T$ or $\delta_{w} T$ could play a role in enhancing $\delta_{C P}$ the necessary eighteen orders of magnitude during baryogenesis. Attempts in this direction have been made in ref. [45], but the existence of these enhancements remains unconvincing. It is most likely that any model of baryogenesis requires new sources of $C P$ violation beyond the standard model, and that electroweak baryogenesis requires the new $C P$ violation to be at the weak scale.

Incorporating additional $C P$ violation into the standard model requires additional matter fields, and there are a number of relatively simple extensions one can consider. Three examples that have been analyzed in the literature and are discussed below are the singlet Majoron model with complex phases in the neutrino mass matrix; the nonsupersymmetric two Higgs doublet model with a $C P$ violating relative phase between the doublets; and the minimal supersymmetric standard model (MSSM) with a $C P$ violating phase in the gaugino masses. In each of these models $\delta_{C P}$ can be $10^{-2}$ or larger, not being suppressed by small couplings as in the KM model, and without being in conflict with experimental limits on the electric dipole moments (EDM) of the electron or neutron. 


\subsection{Timescales}

Having assembled the ingredients is not enough to make a universe: we still require a recipe. The recipe involves the three distinct time scales governing the relevant reactions. These are the thermalization time $\tau_{T}$ which characterizes how fast particles in the cosmic plasma equilibrate; the Higgs time scale $\tau_{H}$ which governs the departure from equilibrium, given by $\tau_{H} \simeq \phi / \dot{\phi}$ as measured by a comoving observer while the expanding bubble wall passes through her; and the sphaleron time $\tau_{s p}$ which governs the rate of baryon violation in the symmetric phase日.

Since baryon violation in the broken phase within the bubbles must be very slow to prevent re-equilibration of the baryon number, we will assume here that baryon number is exactly conserved inside the bubbles. Outside the bubbles in the symmetric phase, $\tau_{s p}$ at the transition temperature $T$ is estimated as

$$
\tau_{s p}^{-1} \simeq \alpha_{w}^{4} T=1.3 \times 10^{-6} T
$$

In contrast, the thermalization rate due to weak and strong interactions, defined for the different particles as the inverse mean free path $\ell_{T}$, is much faster, not having the $\alpha_{w}^{4}$ suppression. It is estimated from strong or weak Coulomb scattering cross sections: [84]

$$
\tau_{T}^{-1} \equiv \ell_{T}^{-1} \simeq\left\{\begin{array}{ll}
0.25 T & \mathrm{q} \\
0.08 T & \mathrm{~W}, \mathrm{Z}, \ell
\end{array} .\right.
$$

These rates correspond to "fast" reactions; some standard processes are much slower, of course, such as chemical equilibration between the first and third families, or between the two chiralities of a light fermion.

The Higgs time scale is less easily determined, and is quite model dependent, roughly given by

$$
\tau_{H}^{-1}=\dot{\phi} / \phi \simeq \frac{v_{w}}{\delta_{w}} \simeq(0.01-1.0) T .
$$

We see that baryon violation is always out of equilibrium near the wall, since $\tau_{s p} \gg \tau_{H}$. However, other particle interactions may or may not be able to equilibrate near the bubble wall, depending on the relative size of $\tau_{H}$ and $\tau_{T}$. This gives rise to two distinct regimes:

1. The adiabatic regime: $\tau_{T} \ll \tau_{H}$. "Fast" interactions maintain thermal equilibrium as the bubble wall passes by and the value of the Higgs field changes. This allows one to

4 In principle there is a fourth time scale, the age of the universe $T / \dot{T}=H^{-1}$, but at the weak epoch it is many orders of magnitude larger than the others and not relevant for microphysics. 
to describe the plasma within the bubble wall in terms of equilibrium thermodynamics with quasistatic chemical potentials for quantities that equilibrate slowly compared to $\tau_{H}$. In this regime baryogenesis occurs within the bubble wall itself.

2. The nonadiabatic regime: $\tau_{T} \gg \tau_{H}$. The wall is thin compared to the mean free path and particles reflect off the oncoming wall with calculable $C P$ violating reflection coefficients. Baryogenesis occurs in an extended region preceding the phase boundary and is enhanced relative to the adiabatic scenario.

Which regime one is in depends on the bubble wall thickness and velocity: for thick or slow walls it is the adiabatic regime that is relevant; for thin or fast walls it is the nonadiabatic.

\subsection{The adiabatic "thick wall" regime: spontaneous baryogenesis}

In the adiabatic limit it should be valid to treat the plasma in the bubble wall as being in quasi-static thermal equilibrium with a classical, time dependent field. The plasma will not be in chemical equilibrium, however, as some reactions, such as baryon violation, are slower than $\tau_{H}^{-1}$. This deviation from chemical equilibrium may be treated by introducing chemical potentials for the slowly varying quantities.

Baryogenesis arising from slowly varying classical Higgs fields during a phase transition is called "spontaneous baryogenesis"; it was first discussed in refs. [128, [1] in the context of second order phase transitions, for which the assumption of adiabaticity is always valid (except in the extremely early universe). To see how time dependent Higgs fields can drive baryon production consider a toy model with a conserved $U(1)_{B}$ symmetry carried by fermions $\psi(B=1), \chi(B=-1)$, a scalar $\phi(B=2)$, and the Yukawa interaction $\bar{\psi} \phi \chi$. During a phase transition in which $\phi$ takes on the classical value

$$
\phi(t)=\rho(t) e^{i \theta(t)}
$$

this leads to baryon production, even for $\dot{\phi}$ small compared to the fermion masses. To see this, redefine the fermion fields by the phase $e^{i \theta(t) B / 2}$; this removes the phase from the Yukawa interaction, but leads to a new interaction from the fermion kinetic term:

$$
\mathcal{L}_{K . E .} \rightarrow \mathcal{L}_{K . E .}+\frac{1}{2} \dot{\theta}\left(\bar{\psi} \gamma^{0} \psi-\bar{\chi} \gamma^{0} \chi\right)=\mathcal{L}_{K . E .}+\frac{1}{2} \dot{\theta} n_{B}
$$

Thus $\dot{\theta}$ acts like a chemical potential for baryon number. (We use the word "charge potential" for $\dot{\theta}$ instead of chemical potential, since the effect is dynamical and not arising from a 
constraint.) The interaction (4.6) splits the energy levels of baryons versus antibaryons so that the free energy is minimized at nonzero baryon number. The interaction (4.6) violates $C$ and $C P$ and will spatially average to zero except in theories with explicit violation of these symmetries.

If $B$ symmetry is violated in some other sector of the theory $(e . g .$, through the electroweak anomaly), then the interaction (4.6) will cause $B$ to try to equilibrate to the value $n_{B}=\mathcal{O}\left(\dot{\theta} T^{2}\right)$. If the $B$ violating interactions are rapid compared to $\ddot{\theta} / \dot{\theta}$ then this equilibrium value will be attained. In the real case we wish to study, however, the rate per unit volume of anomalous fluctuations $\Gamma_{a}$ ( see eq. (2.9)) is too slow to equilibrate due to the factor of $\alpha_{w}^{4}$. In this case one simply integrates $\dot{n}_{B}$ in eq. (4.1) over the appropriate timescale. It should be emphasized that it is not necessary for the charge potential to couple to the baryon current as in the toy example (4.6) in order for there to be a nonzero value for $\dot{n}_{B}$. As long as the charge $X$ is not orthogonal to $B$, an interaction coupling of the form $\dot{\theta} j_{X}^{0}$ will give rise to a nonzero value for $\mu_{B}$ of the form

$$
\mu_{B}=\mathcal{N} \dot{\theta}
$$

where $\mathcal{N}$ is a calculable, model dependent constant.

After the bubble wall has passed by a given point in space, the charge potential $\dot{\theta}$ returns to zero. If baryon violation is still rapid compared to the cooling rate of the universe, the baryon number produced will re-equilibrate to $B=0$. However, the anomalous fluctuation rate $\Gamma_{a}$ goes rapidly to zero as the Higgs vev turns on, so that the baryon number drops out of chemical equilibrium and remains to the present epoch. By means of the inequality (2.10) we can make a crude estimate of this "cut-off" value of the Higgs vev:

$$
\langle\phi\rangle_{c o} \simeq \frac{7 g T}{2 \pi}=0.7 T .
$$

To find the total baryon number produced during the phase transition, eqs. (4.1), (4.7) may be integrated across the bubble wall:

$$
n_{B}=-\frac{9 \mathcal{N}}{T} \int_{-\infty}^{t_{c o}} \mathrm{~d} t \dot{\theta} \Gamma_{a}(\phi(t)) \simeq-\frac{9 \mathcal{N} \Gamma_{a}}{T} \Delta \theta
$$

where we have estimated the integral by treating $\Gamma_{a}$ as a step function equal to its symmetric phase value for $\langle\phi\rangle \leq\langle\phi\rangle_{\text {co }}$ and vanishing further inside the bubble. The quantity $\Delta \theta$ is then the change in $\theta$ from the symmetric phase to the point in the bubble wall where 
baryon violation effectively shuts off; it is a homogeneous function of the $C P$ violating parameter $\delta_{C P}$ of the theory in question. Furthermore, $\Delta \theta$ is homogeneous in $\langle\phi\rangle_{\text {co }}$ and could be very small if $\langle\phi\rangle_{c o}$ proves to be as small as suggested in refs. [51,103].

Given that the entropy density is $s=\left(2 \pi^{2} g_{*} / 45\right) T^{3}$ and $\Gamma_{a}=\kappa\left(\alpha_{w} T\right)^{4}$, one finds for the final baryon to entropy ratio

$$
\frac{n_{B}}{s}=-\kappa\left(\frac{\mathcal{N}}{0.1}\right)\left(\frac{100}{g_{*}}\right)\left(\frac{\Delta \theta}{\pi}\right) \times\left(3 \times 10^{-7}\right) \quad \text { (adiabatic) },
$$

to be compared to the observed value $n_{B} / s \simeq 10^{-10}$. In the above expression, the quantity $\mathcal{N}$ defined in eq. (4.7) is typically $0.1-1.0 ; \kappa$ is the prefactor appearing in equation (2.9) and is thought to be $\mathcal{O}(1) ;\left(100 / g_{*}\right)$ is also close to unity at the weak phase transition. The reason why this result is a formula for the baryon density of the entire universe, even though baryon production is basically a surface effect only occuring within the bubble wall, is that the entire universe is swept out by the bubbles during the course of the phase transition [48].

We now list the steps that must be followed when analyzing weak scale baryogenesis models in the adiabatic limit, and then proceed to give several examples.

(i) Identify a model that has a first order phase transition with large timescale $\tau_{H}$ and classical Higgs field evolution during the phase transition such that there is a $C P$ violating, time dependent phase $\theta(t)$ in the Yukawa interactions.

(ii) Determine $\Delta \theta$ from the Higgs equation of motion in order to use eq. (4.10) to compute the final baryon number. Using the generous estimate (4.8) for $\langle\phi\rangle_{c o}, \Delta \theta$ will typically be $\mathcal{O}(1) \times \delta_{C P}$. Additional suppression is possible if baryon violation shuts off closer to the outer surface of the bubble and $\langle\phi\rangle_{\text {co }}$ proves to be much smaller than assumed.

(iii) Rotate the fermions in the theory to remove the time dependent phase from the Yukawa interactions into a derivative coupling of the form $\dot{\theta} j_{X}^{0}$. The current $X$ is ambiguous, since the rotation is not unique - one can always rotate so that $X \rightarrow$ $(X+Q)$, where $Q$ is a classically conserved charge. It is simplest to choose $X$ so that the rotation is anomaly-free, so one need not treat $\theta(t) W \tilde{W}$ couplings.

(iv) Compute the dynamically generated $\mu_{B}$. To do so one must introduce chemical potentials for each conserved and appoximately conserved quantum number. All the 
particle densities may be determined in terms of these chemical potentials and the charge potential $\dot{\theta}$; for particle species $i$ the density $n_{i}$ is given by

$$
n_{i}=k_{i}\left[X_{i} \dot{\theta}+\sum_{a} \mu_{a} q_{i}^{a}\right] \frac{T^{2}}{6},
$$

where $k_{i}$ is a statistical factor accounting for effective degrees of freedom and differs for fermions and bosons, $X_{i}$ is the $X$ charge of species $i$, and the sum is over all charges out of chemical equilibrium (including $B$ ). The values of the chemical potentials $\mu_{a}$ are fixed by requiring that the primordial plasma not carry any quantum numbers. One can then determine $\mu_{B}$ and the factor $\mathcal{N}$ in eq. (4.7).

(v) Use the expression (4.10) to give the final baryon to entropy ratio. This formula is only expected to be valid deep in the adiabatic thick-wall regime, however. If the equilibration time for particle distributions is long compared with $\tau_{H}$ there may be additional supression factors.

We now present two examples of standard model extensions and show how spontaneous baryogenesis proceeds in them. The first is the two Higgs doublet model, and the second is the minimal supersymmetric standard model (MSSM).

The two Higgs model. This model was first suggested by McLerran as a model for weak scale baryogenesis in ref. [129] and analyzed by Turok and Zadrozny in ref. [49]; the treatment here will follow our subsequent work [52]. The model is characterized by the Lagrangian

$$
\mathcal{L}=\sum_{\text {fermions }} \bar{\psi} i \not D \psi+\sum_{i=1}^{2}\left|D_{\mu} \phi_{i}\right|^{2}-V\left(\phi_{1}, \phi_{2}\right)+\mathcal{L}_{\text {yuk }}+\mathcal{L}_{\text {gauge }}
$$

where the scalar potential is given by [130]

$$
\begin{aligned}
V\left(\phi_{1}, \phi_{2}\right) & =\lambda_{1}\left(\phi_{1}^{\dagger} \phi_{1}-v_{1}^{2}\right)^{2}+\lambda_{2}\left(\phi_{2}^{\dagger} \phi_{2}-v_{2}^{2}\right)^{2}+\lambda_{3}\left[\left(\phi_{1}^{\dagger} \phi_{1}-v_{1}^{2}\right)+\left(\phi_{2}^{\dagger} \phi_{2}-v_{2}^{2}\right)\right]^{2} \\
& +\lambda_{4}\left[\left(\phi_{1}^{\dagger} \phi_{1}\right)\left(\phi_{2}^{\dagger} \phi_{2}\right)-\left(\phi_{1}^{\dagger} \phi_{2}\right)\left(\phi_{2}^{\dagger} \phi_{1}\right)\right]+\lambda_{5}\left[\operatorname{Re}\left(\phi_{1}^{\dagger} \phi_{2}\right)-v_{1} v_{2} \cos \xi\right]^{2} \\
& +\lambda_{6}\left[\operatorname{Im}\left(\phi_{1}^{\dagger} \phi_{2}\right)-v_{1} v_{2} \sin \xi\right]^{2}
\end{aligned}
$$

The Yukawa interactions in $\mathcal{L}_{\text {yuk }}$ couple the $\phi_{1}$ field to up-type quarks; down-type quarks and charged leptons may be coupled exclusively to either $\phi_{1}$ or $\phi_{2}$ - either choice suppresses flavor changing neutral currents and is protected by a discrete symmetry that is softly 
broken. For convenience, we will assume that all fermion masses arise due to interactions with $\phi_{1}$; the other option works equally well, since only the top quark Yukawa interaction proves to be relevant. In either case we can adjust the phases of the Higgs doublets so that, in the fermion mass eigenstate basis, the top quark Yukawa interaction $y_{t}$ is real and $\phi_{1}$ develops a real expectation value at zero temperature. In this basis, aside from the usual KM phase, there is an additional $C P$ violating phase $\xi$ in the scalar potential, so that the zero temperature vev of the fields have a relative phase $e^{i \xi}$ between them. During the weak phase transition the neutral components of the two doublets will acquire time dependent classical values with $\phi_{1}^{0}(t)=\rho(t) e^{i \theta(t)}$ (in unitary gauge where the linear combination of the phases of $\phi_{1,2}^{0}$ eaten by the $Z$ has been rotated away). The change in $\theta$ in going from the beginning of the phase transition to $T=0$ is 52

$$
\Delta \theta=\mathcal{O}\left(\xi-\arctan \left[\frac{\lambda_{6}}{\lambda_{5}} \tan \xi\right]\right)
$$

$\Delta \theta$ will be smaller in going from the symmetric side of the domain wall to the point where the scalar vev equals $\langle\phi\rangle_{c o}$ and baryon violation turns off, since $\Delta \theta$ scales like $\left(\langle\phi\rangle_{c o} / v\right)^{2}$, arising from the change in relative importance between the $\phi^{2}$ and $\phi^{4}$ terms in the potential (4.13).

Next we remove $\theta$ from the Yukawa interactions by performing a time dependent rotation of the fermion fields in the Lagrangian. Since we do not wish to complicate our task and induce any coupling of $\theta$ to gauge fields, we eliminate $\theta$ by means of an anomaly free fermion rotation; thus we rotate the fermions by an amount proportional to their hypercharge. We find that $\theta$ now has derivative couplings to twice the fermionic part of the hypercharge current (the " $X$ " current in this model)

$$
\begin{aligned}
\mathcal{L} & =\mathcal{L}_{\text {K.E. }}-V\left(\phi_{1}, \phi_{2}\right)+y_{t} \bar{q}_{L} \tilde{\rho}_{1} t_{R}+y_{b} \bar{q}_{L} \rho_{1} b_{R}+\ldots+\text { h.c. } \\
& +2 \partial_{\mu} \theta\left[\frac{1}{6} \bar{q}_{L} \gamma^{\mu} q_{L}+\frac{2}{3} \bar{U}_{R} \gamma^{\mu} U_{R}-\frac{1}{3} \bar{D}_{R} \gamma^{\mu} D_{R}-\frac{1}{2} \bar{\ell}_{L} \gamma^{\mu} \ell_{L}-\bar{E}_{R} \gamma^{\mu} E_{R}\right]
\end{aligned}
$$

where there is an implicit sum over the three families in the current coupling to $\partial_{\mu} \theta$, and $\rho_{1}$ is the radial component of $\phi_{1}$. The quantity $\dot{\theta}$ which is nonzero during the phase transition is then a charge potential which splits fermion-antifermion energy levels proportional to their hypercharge inside the domain walls.

We now must calculate the thermodynamic force $\mu_{B}=\mathcal{N} \dot{\theta}$ responsible for the production of baryon number. To this end we introduce chemical potentials for each quantum number that equilibrates slowly compared to $\tau_{H}$. The relevant ones for this model are 
$B-L, Q$, and $B_{3}-\frac{1}{2}\left(B_{1}+B_{2}\right)$ where $B_{i}$ is the baryon number of the $i^{t h}$ family. (There are other conserved or slowly varying charges, such as $B_{1}-B_{2}$, but their chemical potentials turn out to be irrelevant.) From eq. (4.11) we have determined

$$
\mathcal{N}=\frac{4}{3}\left(\frac{6+n}{25+4 n}\right)
$$

where $n$ is the number of physical charged scalars which are light compared to the temperature $T$; for $n=0, \mathcal{N}=0.3$. Comparing eq. (4.10) with (4.14) and (4.16), we find that a value of $\xi \simeq \mathcal{O}\left(10^{-2}-10^{-3}\right)$ for the $C P$ violating parameter in the theory yields an acceptable value for the final baryon to entropy ratio produced in the phase transition.

Our treatment is justified as long as fermionic hypercharge has time to adjust itself through the top quark mass or Yukawa interaction with thermal Higgs particles. This adjustment can occur via scattering (e.g., $\left.t+g \rightarrow t+\phi_{1}\right)$, scalar bremstrahlung (e.g., $\left.t+q \rightarrow t+q+\phi_{1}\right)$ or coherent top mass oscillations within the domain wall. Comparing with photon bremstrahlung [131] we arrive at a crude estimate giving a fermionic hypercharge equilibration rate of $\tau_{Y}^{-1} \simeq \tau_{T}^{-1} \times\left(\lambda_{t} / \pi\right)^{2} \simeq 0.03 T$, where $\tau_{T}^{-1}$ is the strong interaction thermalization rate (4.3). Thus the above analysis is expected to be valid for $\tau_{H}^{-1}$ at the low end of the range (4.4). For less adiabatic phase transitions there may be an additional suppression of our result proportional to $\left(\lambda_{t} / \pi\right)^{2}$ due to the inability of the plasma to reach complete chemical equilibrium in the time available.

A different treatment of the two Higgs model is found in refs. [49], [50], [64]. They find a contribution to the effective action in an expansion of $m_{t} / T$ of the form

$$
\Delta S=-i \zeta(3) \frac{7}{2}\left(\frac{m_{t}}{\pi v_{1}}\right)^{2} \frac{1}{T^{2}} \int \mathrm{d}^{4} x\left[\left(\mathcal{D}_{i} \phi_{1}^{\dagger} \sigma^{a} \mathcal{D}_{0} \phi_{1}+\text { h.c. }\right] \epsilon^{i j k} F_{j k}^{a}\right.
$$

which is argued to bias anomalous baryon violating transitions when the Higgs field $\phi_{1}$ takes on the classical value (4.5); the computation is shown in detail in ref. [64], where an estimate of $n_{B} / s \simeq 10^{-10} \times(C P$ violating angles $)$ is given. This computation is subleading in the adiabatic limit, as it is proportional to $\left(\lambda_{t} / \pi\right)^{2}$. Furthermore the effect of this operator is more strongly affected by the value of $\langle\phi\rangle_{\text {co }}$ where baryon violation cuts off, as its effects scale like $\langle\phi\rangle_{c o}^{4}$ [64, while the factor of $\Delta \theta$ in formula (4.10) scales like $\langle\phi\rangle_{c o}^{2}$. 
Minimal Supersymmetry. One of the most attractive extensions of the standard model is the minimal supersymmetric standard model (MSSM) [130]. It possesses two Higgs doublets, but does not follow the above treatment, since supersymmetry ensures that there be no $C P$ violation in the Higgs potential $\left(\lambda_{5}=\lambda_{6}\right)$. Nonetheless, the MSSM has two possible sources of $C P$ violation, the phases conventionally called $\phi_{A}$ and $\phi_{B}$, which are absent in the minimal standard model [132]; a combination of these will contribute to baryogenesis. The interactions in this model are given in terms of superfields by

$$
\begin{aligned}
& {\left[\bar{U} \lambda_{U} Q H+\bar{D} \lambda_{D} Q H^{\prime}+\bar{E} \lambda_{E} L H^{\prime}+|\mu| e^{-i \phi_{B}} H H^{\prime}\right]_{F}} \\
& +m_{3 / 2}\left[|A| e^{i \phi_{A}}\left(\bar{U} \xi_{U} Q H+\bar{D} \xi_{D} Q H^{\prime}+\bar{E} \xi_{E} L H^{\prime}\right)+\left|\mu_{B}\right| H H^{\prime}\right]_{A},
\end{aligned}
$$

along with mass terms for the gauginos. The $C P$ violating phases $\phi_{A}$ and $\phi_{B}$ occur only in interactions involving the super-partners of the ordinary particles.

Suggestions for how spontaneous baryogenesis in the MSSM at the weak phase transition could proceed were first made in refs. [51] and [52]; the first quantitative analysis is given in ref. [55]. There it was found that baryogenesis was negligibly affected by the phase $\phi_{A}$, but proceeded due to the effects of the phase $\phi_{B}$ which appears in the mass matrix of the supersymmetric partners of the gauge bosons and the Higgs scalars (the "inos").

The generalization of spontaneous baryogenesis from a single fermion to several fermions with a spacetime dependent mixing involves making a space-time dependent unitary change of basis on the fermions $\psi_{i} \rightarrow U_{i j}\left(x_{\mu}\right) \psi_{j}$ in order to render the fermion mass terms everywhere real, positive and diagonal; the space-time dependence of $U$ requires that we replace the kinetic energy terms in the Lagrangian by

$$
\mathcal{L}_{\text {K.E. }} \rightarrow \mathcal{L}_{\text {K.E. }}+\bar{\psi} \gamma^{\mu}\left(U^{\dagger} i \partial_{\mu} U\right) \psi .
$$

It is simplest to choose $U$ to be an anomaly free rotation, so that there are no $W \tilde{W}$ terms generated, which forces $\left(U^{\dagger} i \partial_{\mu} U\right)$ to couple to ordinary quarks as well as inos. In ref. [55] it is shown that the generalization of eq. (4.9) is

$$
n_{B}=\frac{3}{T} \int_{0}^{\langle\phi\rangle_{c o}} d \phi \operatorname{Tr} U^{\dagger}(\phi) \frac{i d U(\phi)}{d \phi} t_{\mathrm{wk}}^{2} \Gamma_{a}(\phi),
$$

where $\langle\phi\rangle_{c o}$ is the value of the Higgs vev for which $\Gamma_{a}$ starts falling exponentially (see eq. (4.8)), and $t_{\mathrm{wk}}^{2}$ is the $S U(2)$ Casimir matrix. Eq. (4.20) leads to an acceptable value for $n_{B} / s$ for certain regions of parameter space in the MSSM. However, between the restrictions that the phase transition be sufficiently first order and that $n_{B} / s \simeq 10^{-10}$, a large region of parameter space is excluded, and the theory is experimentally testable as a baryogenesis candidate, a luxury accorded to few models. 


\subsection{The nonadiabatic "thin wall" regime: the charge transport mechanism}

Thin bubble walls occur naturally in models with scalar fields with trilinear couplings (e.g., a familiar extension of the MSSM), as well as in the two Higgs model or singlet Majoron with some tuning of parameters. For bubble walls thinner than the strong interaction mean free path (4.3), $\delta_{w}<\ell_{T} \simeq 4 / T$, incoming fermions in the bubble wall frame interact with the wall like quantum mechanical particles scattering from a potential barrier. Due to $C P$ violating interactions with the scalar field, the reflection coefficients for the fermions can be such that a net charge is reflected from the domain wall, which is transported into the region preceding the bubble. This charge asymmetry (not necessarily in baryon number) can bias anomalous $S U(2)$ fluctuations in a large region in front of the bubble wall and produce a baryon asymmetry. This "charge transport mechanism" was introduced in the context of the singlet Majoron model, where the fermions scattering of the wall were massive neutrinos [48]; here we will summarize ref. [53] on the nonadiabatic limit of the two Higgs model discussed above. Potentially the most compelling application of the nonadiabatic regime would be the MSSM extended by a singlet field [130], but that model has not been analyzed to date.

The procedure that has been developed for analyzing the thin wall scenario is as follows:

(i) Compute the wall velocity and profile, including the space dependent $C P$ violating phase, from the finite temperature effective potential.

(ii) Calculate the reflection coefficients for fermions striking the wall with momentum $\vec{k}$ in the domain wall frame of reference by integrating the Dirac (Majorana) equation of motion. Because of $C P$ violation, the reflection probabilities for particles and antiparticles can be different.

(iii) Convolute the reflection probabilities with the incoming (boosted) thermal flux of particles to determine the flux of fermions reflected from the wall. Due to $C P$ violation, this flux will carry nonzero quantum numbers $X$.

(iv) The nonzero $X$ flux from the wall will lead to a nonzero charge density $n_{X}$ in the region preceding the wall. In general, a nonzero charge $X$ will cause the free energy to be minimized for nonzero $B$, and one finds

$$
\mu_{B}=\left.\frac{\partial F}{\partial B}\right|_{B=0}=-\mathcal{C} \frac{n_{X}}{T^{2}},
$$


where $n_{X}$ is a nonzero $X$-charge density. Given eq. (4.1), the baryon density in a region which is a distance $d(t)=\left(z-v_{w} t\right)$ in front of the domain wall equals

$$
\begin{aligned}
n_{B}(z, t) & =-\frac{9 \Gamma_{a}}{T} \int_{-\infty}^{z / v_{w}} \mathrm{~d} t \frac{\partial F}{\partial B}=\mathcal{C} \frac{9 \Gamma_{a}}{T} \int_{-\infty}^{z / v_{w}} \mathrm{~d} t n_{X}\left(z-v_{w} t\right) \\
& =\mathcal{C} \frac{9 \Gamma_{a}}{T} \frac{1}{v_{w}} \int_{0}^{\infty} \mathrm{d} z n_{X}(z) .
\end{aligned}
$$

Thus one needs to calculate the total amount of charge in the region in front of the wall in order to compute the net baryon density produced. (As in the adiabatic scenario, baryons that are produced pass into the broken phase and become stable; a major difference though is that the baryon asymmetry is produced in front of the bubble in the symmetric phase, rather than within the bubble wall.)

The applications of this procedure to the two Higgs model is detailed in ref. [53]. Rather than compute the bubble wall parameters from the effective potential, we use a representative wall profile

$$
\phi_{1}(z)=v_{1}\left[\frac{1+\tanh \left(z / \delta_{w}\right)}{2}\right] e^{-i \Delta \theta\left[\tanh \left(z / \delta_{w}\right)-1\right] / 2},
$$

where the top mass $m_{t}(T)=\lambda_{t} v_{1}$ and the wall width $\delta_{w}$ are taken as free parameters to vary, and $\Delta \theta=-\pi$ is assumed; the resultant baryon asymmetry should scale linearly for small $\Delta \theta$. The only fermion that has appreciable reflection probability from the domain wall is the top quark, and $C P$ violation causes an asymmetry in the reflection rate between right-handed and left-handed top quarks, leading to a flux carrying net axial charge shown in fig. 5. The asymmetry is maximized for particles whose longitudinal momentum in the wall frame is of order the top mass in the broken phase at that temperature (all particles get reflected or transmitted, if their longitudinal momenta are lower or much higher than $m_{t}$, respectively). From these $\vec{k}$ dependent reflection probabilities the momentum distribution of the reflected flux is computed. The transport of the axial top quark number in front of the wall is calculated by Monte Carlo techniques, allowing each reflected top quark to undergo Coulomb scattering via gluon exchange (with an infrared cutoff in the form of a plasmon mass) until the wall catches up. The proportionality factor $\mathcal{C}$ in eq. (4.21) is found to equal $4 /(1+2 n)$ in the standard model augmented to have $n$ Higgs doublets, and from eq. (4.22) we compute the baryon asymmetry.

Axial top number is not a good quantum number, as it is violated by Higgs scattering, and so in ref. [53] fermionic hypercharge was identified as the transported charge. In fig. 6] 
we display the results of the Monte Carlo simulation for several parameters, showing a substantial transport of charge in front of the domain wall. The penetration distance of the charge pushed in front of the wall depends on the velocity of the wall, and for $v_{w}=1 / \sqrt{3}$ the sound velocity — we find a distance of $\sim 40 / T$; for slower walls, the distance is greater. There are several factors contributing to this large distance: (i) The incident particles selected by the reflection coefficients have longitudinal momentum $p_{L} \sim m_{t}=2 T$, and so have higher than average energy to start with; (ii) Upon reflection, the particle energies are enhanced by $\gamma(1+\beta) \simeq 2$, for $v_{w}=1 / \sqrt{3}$; (iii) Particles typically experience numerous collisions, but primarily in the forward direction. Our numerical results are consistent with Bjorken's energy loss formula [133] for light quarks in a quark-gluon plasma

$$
\frac{\mathrm{d} E}{\mathrm{~d} x}=-\frac{8 \pi}{3} \alpha_{s}^{2}\left(1+N_{f} / 6\right) T^{2} \ln \sqrt{4 E T / M^{2}},
$$

where $N_{f}=6$ is the number of quark flavors in the plasma and $M$ is an infrared cuttoff $(\simeq$ the plasmon mass): this formula yields a stopping distance of $\ell \simeq\left(9 / 8 \pi \alpha_{s}^{2}\right) T^{-1}=35 T^{-1}$ for a reflected top quark with initial energy $E \simeq 6 T$, twice the average thermal energy. (iv) Finally, for very slow domain walls, diffusion of charge in front of the wall plays a large role and increases the penetration distance substantially.

Khlebikhov has shown by means of the Vlasov equations that the above scenario cannot proceed exactly as described above, since hypercharge is gauged and any hypercharge density produced will be screened by the plasma [134]. In ref. [54] this error was corrected. The correct charge to consider as the " $X$ " charge in eq. (4.22) is not fermionic hypercharge $Y_{f}$, but rather the linear combination $B^{\prime}=B-x Y_{f}$ where $B$ is baryon number and $x$ is chosen so that $B^{\prime}$ is not screened by gauge forces; we find $x=2 /(10+n)$, where $n$ is the number of scalar doublets. The reflected flux of top quarks carries $B^{\prime}$ charge, and upon redoing the analysis of ref. [53], the final results for the produced baryon asymmetry are found to be unchanged. These are shown in fig. 7 . The contour plot shows that a very large baryon asymmetry can result for maximal $C P$ violation, $v_{w}=1 / \sqrt{3}$ and $\delta_{w} \times m_{t}=\mathcal{O}(1)$, where $m_{t}$ is the value of the top mass in the broken phase at the phase transition. For a wall velocity $v_{w}=0.1$ one finds an order of magnitude enhancement in $n_{B} / s$ [53].

We conclude that for thin bubble walls the charge transport mechanism works very well for producing the baryon asymmetry, and in fact suffers from fewer theoretical uncertainties than the adiabatic scenarios where baryogenesis actually takes place within the bubble wall. 


\subsection{Conclusions about mechanisms}

As is evident from the above discussion, the mechanism for weak scale baryogenesis depends on the width and velocity of the bubble wall. Allowing for certain processes to remain in thermal and chemical equilibrium during the phase transition, we have been able to show how weak scale baryogenesis could proceed in both the adiabatic and nonadiabatic regimes, producing a phenomenologically acceptable value for $n_{B} / s$. It is tempting to "interpolate" and assume that baryogenesis would also work for the interesting case of phase transitions that lie between these two regimes, where scattering lengths are comparable to interaction lengths with the domain wall; however, the tools of equilibrium thermodynamics do not suffice for such a regime which would have to be analyzed by means of the Boltzman equation. Such a treatment could afford detailed knowledge about the crucial departure from equilibrium in all regimes.

In addition to the models discussed above there are a number of others in the literature, which we include for completeness. An early model of McLerran 129 attempted to use cosmological axions to provide the required $C P$ violation in standard model baryogenesis, but the resultant baryon asymmetry proved to be too small. Other weak scale baryogenesis investigations include the effect of anomalous QCD events at the weak scale [135]; leftright models [136, 137]; transient scalar-top condensation in supersymmetric models [138]; lepton number production from domain walls colliding at the end of the transition [139]; and baryogenesis from electroweak strings without a first order phase transition [140.

\section{CONSTRAINTS AND EXPERIMENTAL SIGNATURES}

One of the most appealing features of weak scale baryogenesis is the implication that new physics lies in wait for us at the weak scale. Since weak scale baryogenesis is feasible in most extensions of the standard model experimental guidance is needed.

Possible and expected experimental consequences of weak scale baryogenesis are:

1. New particles. The requirement that the weak phase transition be sufficiently first order, combined with Higgs mass limits from LEP, imply that the weak symmetry breaking sector be extended beyond a single Higgs doublet. Furthermore, the requirement that there be new $C P$ violation also requires that new particles be added to the standard model. Both constraints suggest that there ought to be more than one scalar particle for the SSC to find. 
2. New $C P$ violation. The new sources of $C P$ violation may be large enough to see in future experiments measuring the electric dipole moment of the electron and neutron [141], atomic physics [142], or top quark production [143].

3. Constraints on supersymmetry. Spontaneous baryogenesis at the weak scale in the MSSM puts significant constraints on the experimentally allowed region of parameter space. For example the minimal supersymmetric standard model can avoid baryon number washout provided that the lightest Higgs scalar is lighter than about $50 \mathrm{GeV}, \tan \beta$, the ratio of the two Higgs vevs, is smaller than about 1.7, the top quark is heavier than about $150 \mathrm{GeV}$, and the squarks are lighter than about $150 \mathrm{GeV}$ [106]. (All of these bounds are only approximate because they are computed in one loop perturbation theory, and can be weakened by allowing the top squark to be significantly lighter than the other squarks.) Creating sufficient baryon asymmetry requires the electric dipole moment of the neutron to be greater than $10^{-27} \mathrm{e}-\mathrm{cm}[55]$.

4. Flavor changing neutral currents. Typical extensions of the standard model that are appropriate for baryogenesis (e.g.the two Higgs model) could have significant flavor changing neutral currents large enough to see in the heavy flavor sector. Interesting places to look are $B^{0} \rightarrow \mu^{+} \mu^{-}$[144], $t \rightarrow c \gamma$, and $t \rightarrow c Z$ [145].

5. Dark matter searches. Anomalous weak processes could explain the relative abundance of dark matter to baryon number in the universe today by coproduction of massive stable particles along with baryons through the anomaly [146]. These particles would have to carry weak interactions, and are tightly constrained by present dark matter searches [147].

\section{FUTURE CALCULATIONS}

We have seen that the baryon number of the universe may have been produced at the weak phase transition via experimentally accessible weak scale physics. A precise calculation of the baryon abundance produced in a given model would allow us to pin down the parameters in that model, much as the abundances of the light elements and of dark matter are used to constrain models. So far, the baryogenesis calculations have been qualitative, and probably only accurate to within a couple of orders of magnitude. A more quantitative calculation will require further analytic and numerical work on the following questions: 
1. A lattice study of the order and parameters (critical temperature, value of the order parameter at the critical temperature) of the electroweak phase transition. It is is of particular interest to see how the parameters depend on the Higgs mass.

2. A computation of the baryon violation rate in both phases and within the bubble wall itself.

3. A quantitative calculation of the nonequilibrium transport of quantum numbers is needed both for all but the most adiabatic cases, for instance by numerically solving the Boltzmann equations.

\section{Acknowledgements}

The work of A.N. and D.K. was supported in part by the Department of Energy under contract \#DE-FGO3-90ER40546 and fellowships from the Alfred P. Sloan Foundation. D.K. is also supported in part by the NSF under contract PHY-9057135. The work of A.C. was supported in part by the Department of Energy under contract \#DE-FG0291ER40676 and by the Texas National Research Laboratory grant \#RGFY92B6. 


\section{References}

[1] Kolb EW, Turner MS, The Early Universe, Addison-Wesley (1990)

[2] Sakharov AD, JETP Lett.5:24 (1967)

[3] Yoshimura M, Phys. Rev. Lett. 41:281 (1978); E Phys. Rev. Lett. 42:7461 (1979)

[4] Dimopoulos S, Susskind L, Phys. Rev. D18:4500 (1978)

[5] Ignatiev AYu, Krasnikov NV, Kuzmin VA, Tavhelidze AN, Phys. Lett. 76B:436 (1978)

[6] Toussaint D, Treiman S, Wilczek F, Zee A, Phys. Rev. D19:1036 (1979)

[7] Weinberg S, Phys. Rev. Lett. 42:850 (1979)

[8] Nanopoulos DV, Weinberg S, Phys. Rev. D20:2484 (1979)

[9] Barr SM, Segre G, Weldon HA, Phys. Rev. D20:2494 (1979)

[10] Georgi H, Glashow S, Phys. Rev. Lett. 32:438 (1974)

[11] t'Hooft G, Phys. Rev. Lett. $37: 8$ (1976); Phys. Rev. D14:3432 (1976)

[12] Linde AD, Phys. Lett. 70B:306 (1977)

[13] Manton NS, Phys. Rev. D28:2019 (1983); Klinkhammer FR, Manton NS, Phys. Rev. D30:2212 (1984)

[14] Forgacs P, Horvath Z, Phys. Lett. 138B:397 (1984)

[15] Kuzmin VA, Rubakov VA, Shaposhnikov ME, Phys. Lett. 155B:36 (1985)

[16] Arnold P, McLerran L, Phys. Rev. D36:581 (1987); Phys. Rev. D37:1020 (1988)

[17] Khlebnikov SYu, Shaposhnikov ME, Nucl. Phys. B308:885 (1988)

[18] Ambjorn J, Laursen M, Shaposhnikov M, Phys. Lett. 197B:49 (1989); Ambjorn J, Askaard T, Porter H, Shaposhnikov ME, Phys. Lett. 244B:479 (1990); Nucl. Phys. B353:346 (1991); Ambjorn J, Farakos K, Phys. Lett. 294B:248 (1992)

[19] Carson L, Li X, McLerran L, Wang RT, Phys. Rev. D42:2127 (1990)

[20] Dine M, Lechtenfeld O, Sakita B, Fischler W, Polchinski J, Nucl. Phys. B342:381 (1990)

[21] Aoki K-I, Phys. Lett. 174B:371 (1986)

[22] Kolb EW, Turner MS, Mod. Phys. Lett. A2:285 (1987)

[23] Kuzmin VA, Rubakov VA, Shaposhnikov ME, Phys. Lett. 191B:171 (1987)

[24] Harvey JA, Turner MS, Phys. Rev. D42:3344 (1990)

[25] Nelson AE, Barr SM, Phys. Lett. 246B:141 (1990)

[26] Dolgov AD, Phys. Rep. 222:309 (1992)

[27] Fukugita M, Yanagida T, Phys. Rev. D42:1285 (1990)

[28] Campbell BA, Davidson S, Ellis F, Olive K, Phys. Lett. 256B:457 (1991)

[29] Fischler W, Giudice GF, Leigh RG, Paban S, Phys. Lett. 258B:45 (1991)

[30] Zel'dovich YaB, Khlopov MYu, Phys. Lett. 79B:239 (1978)

[31] Preskill J, Phys. Rev. Lett. 54:1365 (1979)

[32] Dimopoulos S, Georgi H, Nucl. Phys. B193:150 (1981)

[33] Sakai N, Z. Phys. C11:153 (1981) 
[34] Weinberg S, Phys. Rev. Lett. 48:1303 (1982)

[35] Ellis J, Linde AD, Nanopoulos DV, Phys. Lett. 118B:59 (1982)

[36] Krauss LM, Nucl. Phys. B227:556 (1983)

[37] Khlopov MYu, Linde AD, Phys. Lett. 138B:265 (1984)

[38] Ellis J, Kim JE, Nanopoulos DV, Phys. Lett. 145B:181 (1984)

[39] Ovrut BA, Steinhardt PJ, Phys. Lett. 147B:263 (1984)

[40] Guth AH, Phys. Rev. D23:347 (1981)

[41] Linde AD, Rep. Prog. Phys. 42:389 (1979) ; Phys. Lett. 108B:389 (1982)

[42] Albrecht A, Steinhardt PJ, Phys. Rev. Lett. 48:1220 (1982)

[43] Kirzhnitz DA JETP Lett. 15:529 (1972); Kirzhnitz DA, Linde AD, Phys. Lett. 72B:471 (1972)

[44] Kobayashi M, Maskawa M, Prog. Theor. Phys. 49:652 (1973)

[45] Shaposhnikov ME, JETP Lett. 44:364 (1986); Nucl. Phys. B287:757 (1987); Nucl. Phys. B299:797 (1988); Phys. Lett. 277B (1992) 324, Erratum, Phys. Lett. 282B (1992) 483

[46] Ambjorn J, Farakos K, Shaposhnikov ME, Niels Bohr Institute preprint NBI-92-20 (1992)

[47] Jarlskog C, Z.C. Physik 29:491 (1985), Phys. Rev. Lett. 55:1039 (1985)

[48] Cohen AG, Kaplan DB, Nelson AE, Phys. Lett. 245B:561 (1990); Nucl. Phys. B349:727 (1991)

[49] Turok N, Zadrozny J, Phys. Rev. Lett. 65:2331 (1990); Nucl. Phys. B358:471 (1991)

[50] McLerran L, Shaposhnikov M, Turok N, Voloshin M, Phys. Lett. 256B:451 (1991)

[51] Dine M, Huet P, Singleton R, Susskind L, Phys. Lett. 257B:351 (1991)

[52] Cohen AG, Kaplan DB, Nelson AE, Phys. Lett. 263B:86 (1991)

[53] Nelson AE, Kaplan DB, Cohen AG, Nucl. Phys. B373:453 (1992)

[54] Cohen AG, Kaplan DB, Nelson AE, Phys. Lett. 294B:57 (1992)

[55] Cohen AG, Nelson AE, Phys. Lett. 297B:111 (1992)

[56] Chikashige Y, Mohapatra R, Peccei R, Phys. Lett. 98B:265 (1981)

[57] Coleman S, Aspects of Symmetry, Cambridge (1985)

[58] Langer J, Ann. Phys. 54:258 (1969); Physica 73:61 (1974)

[59] Affleck I, Phys. Rev. Lett. 46:388 (1981)

[60] Linde AD, Phys. Lett. 93B:327 (1980)

[61] Gross DJ, Pisarski RD, Yaffe L, Rev. Mod. Phys. 53:1 (1981)

[62] Grigoriev D, Rubakov V, Shaposhnikov M, Phys. Lett. 216B:172 (1989);

Nucl. Phys. B326:737 (1989)

[63] Liu B, McLerran L, Turok N, Phys. Rev. D46:2668 (1992)

[64] Turok N, Imperial preprint TP-91-92-33 (1992)

[65] Grigoriev D, Turok N, Shaposhnikov M, Phys. Lett. 275B:395 (1992) 
[66] Dine M, Proc. 1st Yale-Texas Workshop "Baryon Violation at the Electroweak Scale", ed. Krauss L, Rey S J, (World Scientific 1992)

[67] Coleman S, Weinberg E, Phys. Rev. D7:1888 (1973)

[68] Kirzhnitz DA, Linde AD, Ann. Phys. 101:195 (1976)

[69] Guth AJ, Weinberg EJ, Phys. Rev. Lett. 45:1131 (1980)

[70] Witten E, Nucl. Phys. B177:477 (1981)

[71] Heller UM, Phys. Lett. 191B:109 (1987)

[72] Weinberg EJ, Wu A, Phys. Rev. D36:2474 (1987)

[73] Symanzik K, Comm. Math. Phys. 16:48 (1970)

[74] Iliopoulos J, Itzykson C, Martin A, Rev. Mod. Phys. 47:165 (1975)

[75] Iliopoulos J, Papanicolaou N, Nucl. Phys. B111:209 (1976)

[76] Kondo Y, Umemura I, Yamamoto Y, Phys. Lett. 263B:93 (1991)

[77] Dolan J, Jackiw R, Phys. Rev. D9:3320 (1974)

[78] Weinberg S, Phys. Rev. D9:3357 (1974)

[79] Kirzhnitz DA, Linde AD, JETP 40:628 (1974)

[80] Bochkarev AI, Khlebnikov SYu, Shaposhnikov ME, Nucl. Phys. B329:490 (1990)

[81] Anderson GE, Hall LJ, Phys. Rev. D45:2685 (1992)

[82] Turok N, Phys. Rev. Lett. 68:1803 (1992)

[83] Carrington ME, Phys. Rev. D45:2933 (1992); University of Minnesota preprint WIN92-06, (1992)

[84] Dine M, Leigh RL, Huet P, Linde L, Linde D, Phys. Lett. 283B:319 (1992); Phys. Rev. D46:550 (1992)

[85] Arnold P, Phys. Rev. D46:2628 (1992)

[86] Boyd CG, Brahm DE, Hsu SDH, University of Chicago preprint EFI-92-22 (1992)

[87] Arnold P, Espinosa E, University of Washington preprint UW/PT-92-18 (1992)

[88] Bagnasco JE, Dine M, UCSC preprint SCIPP-92-43 (1992)

[89] Mori T, "Searches for the neutral Higgs Boson at LEP", Talk given at the XXVI Rochester International Conference on High Energy Physics, Dallas, Texas August 6-12, (1992)

[90] Gleiser M, Kolb EW, Watkins R, Nucl. Phys. B364:411 (1991)

[91] Tetradis N, DESY preprint DESY-91-151, (1991)

[92] Gleiser M, Kolb EW, Phys. Rev. Lett. 69:1304 (1992)

[93] Gleiser M, Ramos RO, Dartmouth preprint DART-HEP-92-08, (1992)

[94] Jansen K, Seuferling P, Nucl. Phys. B343:507 (1990)

[95] Gavai RV, Heller UM, Karsch F, Neuhaus T, Plache B Nucl. Phys. B (proc. suppl.)26:539 (1992); Phys. Lett. 294B:84 (1992)

[96] Tetradis N, Wetterich C, DESY preprint 92-093 (1992)

[97] Evertz HG, Jersak J, Kanaya K, Nucl. Phys. B285:229 (1987)

[98] Jain V, MPI preprint MPI-PH/92-72 (1992) 
[99] Jain V, Papadopoulos A, LBL preprint 33067 (1992)

[100] Ginsparg P, Nucl. Phys. B170:388 (1980)

[101] March-Russell J, Phys. Lett. 296B:364 (1992)

[102] Bochkarev A, Kuzmin S, Shaposhnikov M, Phys. Lett. 244B:27 (1990)

[103] Dine M, Huet P, Singleton B, Nucl. Phys. B375:625 (1992)

[104] Turok N, Zadrozny J, Nucl. Phys. B369:729 (1992)

[105] Giudice GF, Phys. Rev. D45:3177 (1992)

[106] Myint S, Phys. Lett. 287B:325 (1992)

[107] Land D, Carlson ED, Phys. Lett. 292B:107 (1992)

[108] Sei N, Umemura I, Yamamoto K, Kyoto preprint NEAP-49 (1992)

[109] Enqvist K, Vilja I, Phys. Lett. 287B:119 (1992)

[110] Pietroni M, Padua preprint, DFPD-92-TH-36 (1992)

[111] Damgaard PH, Heller UM, Phys. Lett. 171B:442 (1986); Nucl. Phys. B294:253 (1987); Nucl. Phys. B304:63 (1988)

[112] Bunk B, Ilgenfritz EM, Kripfganz J, Schiller A, Phys. Lett. 284B:371 (1992); preprint BI-TP-92-46 (1992)

[113] Takahashi K, Z. Phys.C26:601 (1985)

[114] Fendley P, Phys. Lett. 196B:175 (1987)

[115] Parwani R, Phys. Rev. D45:4695 (1992)

[116] Espinosa JR, Quiros M, Zwirner F, Phys. Lett. 191B:115 (1992); CERN preprint TH.6577/92 (1992)

[117] Buchmuller W, Heilbig T, DESY preprint 92-117 (1992); Buchmuller W, Heilbig T Walliser D, DESY preprint 92-151 (1992)

[118] Elmfors P, Nordita preprint 92-63-P (1992)

[119] Amelino-Camelia G, Pi S-Y, Boston University preprint BUHEP-92-26 (1992)

[120] Carrington ME, Kapusta JI, University of Minnesota preprint TPI-MINN-92/55-T (1992)

[121] Gelmini G, Gleiser M, UCLA preprint HEP-92-44 (1992)

[122] Anderson G, Phys. Lett. 295B:32 (1992)

[123] Freese K, Kamionkowski M, Phys. Rev. Lett. 69:2743 (1992)

[124] Huet P, Kajantie K, Leigh RG, Liu B-H, McLerran L, SLAC preprint SLAC-PUB-5943 (1992)

[125] Linde AD, Phys. Lett. 100B:37 (1981); Nucl. Phys. B216:421 (1983)

[126] Turok N, Princeton preprint PUPT-91-1273 (1991)

[127] Khlebnikov SYu, Phys. Rev. D46:3223 (1992)

[128] Cohen AG, Kaplan DB, Phys. Lett. 199B:251 (1987); Nucl. Phys. B308:913 (1988)

[129] McLerran L, Phys. Rev. Lett. 62:1075 (1989)

[130] Gunion JF, Haber HE, Kane GL, Dawson S, The Higgs Hunter's Guide, AddisonWesley Publishing Company, (1990) 
[131] Bjorken JD, Drell SD, Relativistic Quantum Mechanics, McGraw-Hill (1965)

[132] Ellis J, Ferrara S, Nanopoulos DV, Phys. Lett. 114B:231 (1982); Buchmuller W, Wyler D, Phys. Lett. 121B:321 (1983); Polchinski J, Wise MB, Phys. Lett. 125B:393 (1983);del Aguila F, Gavela M, Grifols J, Mendez A, Phys. Lett. 126B:71 (1983); Nanopoulos DV, Srednicki M, Phys. Lett. 128B:61 (1983);Dugan M, Grinstein B, Hall LJ, Nucl. Phys. B255:413 (1985)

[133] Bjorken JD, FERMILAB-Pub-82-59-THY (1982); Braaten E, Thoma M, Phys. Rev. D44:R2625 (1991)

[134] Khlebnikov SYu, UC Los Angeles preprint UCLA-92-TEP-14 (1992)

[135] Mohapatra RN, Zhang X, Phys. Rev. D45:2699 (1992)

[136] Mohapatra RN, Zhang X, University of Maryland preprint UMDHEP-92-230 (1992)

[137] Frère J-M, Houart L, Moreno JM, Orloff J, Tytgat M, CERN preprint TH 6747/92 (1992)

[138] Sher M, William \& Mary College preprint WM-92-107 (1992)

[139] Masiero A, Riotto A, Phys. Lett. 289B:73 (1992)

[140] Brandenberger RH, Davis AC, Brown University preprints BROWN-HET-862, BROWNHET-865 (1992)

[141] Barr SM, Marciano WJ, in CP Violation, ed. C. Jarlskog (World Scientific, Singapore, 1989) Bernreuther W, Suzuki M, Rev. Mod. Phys. 63:313 (1991)

[142] Barr SM, Phys. Rev. Lett. 68:1822 (1992); Phys. Rev. D45:4148 (1992)

[143] Atwood D, Soni A, Phys. Rev. D45:2405 (1992), Schmidt CR, Peskin ME, Phys. Rev. Lett. 69:410 (1992); Schmidt CR, Phys. Lett. 293B:111 (1992)

[144] Savage MJ, Phys. Lett. 266B:135 (1991)

[145] Luke M, Savage MJ, UC San Diego preprint UCSD-PTH-93-01

[146] Barr SM, Chivukula SR, Farhi E, Phys. Lett. 241B:387 (1990); Barr SM, Phys. Rev. D44:3062 (1991); Kaplan DB, Phys. Rev. Lett. 68:741 (1992)

[147] Ahlen SP et al., Phys. Lett. 195B:603 (1987); Caldwell DO et al., Phys. Rev. Lett. 61:510 (1988) 


\section{Figure Captions}

Fig. 1. The potential energy of the $S U(2)$ gauge field along the "sphaleron" trajectory. The minima correspond to configurations with zero gauge field strength, but different baryon number. The lower curve ignores the energy of the baryons, while the upper curve does not. The zero of energy for the two curves has been shifted for clarity.

Fig. 2. $N_{C S}$ as a function of time in the Abelian Higgs model. (From [62]).

Fig. 3. The effective potential for a theory which has a first order phase transition. The dotted line is the potential at the critical temperature $T_{c}$, which has two degenerate minima. The solid line is the potential at the low temperature $T_{0}$, where only the broken symmetry phase is stable. The dashed line shows the potential at an intermediate temperature where the broken phase has lowest free energy but the symmetric phase is metastable. The dot-dashed line is the potential at high temperatures, where only the symmetric phase is stable.

Fig. 4. Typical "daisy" contribution to the effective potential

Fig. 5. The difference in reflection probabilities for left and right handed top quarks, as a function of the longitudinal momentum in the wall frame, for three different wall widths. The wall shape is given in (4.23), with $\Delta \theta=-\pi$, corresponding to maximal $C P$ violation.

Fig. 6. A plot of the ratio of hypercharge to entropy densities preceding the bubble wall, as seen in the thermal frame of reference. The horizontal axis is the distance ahead of the wall, in units of the inverse temperature. These computations were performed assuming $m_{t}=2 T$ and $\delta_{w}=1 / T$, for the two sample wall velocities $v_{w}=1 / \sqrt{3}(\gamma=1.225)$ and $v_{w}=.98(\gamma=5.0)$. It is the area under these curves that enters eq. (4.22) for $n_{B}$.

Fig. 7. Contour plot of $n_{B} / s$ as a function of the top mass and $\delta_{w}$, in units of the critical temperature, assuming maximal $C P$ violation $(\Delta \theta=-\pi$ in eq. (4.23)). We have assumed the number of light scalars to be $n=2$, the wall velocity to be $v_{w}=1 / \sqrt{3}$ and have taken $\kappa=1$ in eq. (2.9). 